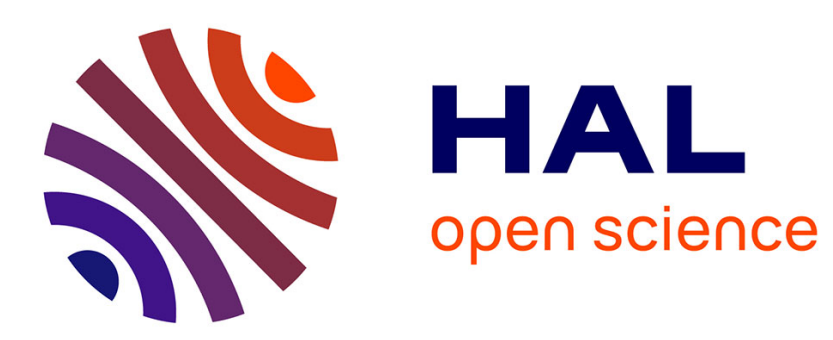

\title{
On asymptotic elastodynamic homogenization approaches for periodic media
}

Hussein Nassar, Qi-Chang He, Nicolas Auffray

\section{To cite this version:}

Hussein Nassar, Qi-Chang He, Nicolas Auffray. On asymptotic elastodynamic homogenization approaches for periodic media. Journal of the Mechanics and Physics of Solids, 2016, 88, pp.274-290. hal-01249157

\section{HAL Id: hal-01249157 \\ https://hal.science/hal-01249157}

Submitted on 30 Dec 2015

HAL is a multi-disciplinary open access archive for the deposit and dissemination of scientific research documents, whether they are published or not. The documents may come from teaching and research institutions in France or abroad, or from public or private research centers.
L'archive ouverte pluridisciplinaire HAL, est destinée au dépôt et à la diffusion de documents scientifiques de niveau recherche, publiés ou non, émanant des établissements d'enseignement et de recherche français ou étrangers, des laboratoires publics ou privés. 


\title{
On asymptotic elastodynamic homogenization approaches for periodic media
}

\author{
H. Nassar, Q.-C. He*, N. Auffray* \\ Université Paris-Est, Modélisation et Simulation Multi-Echelle, MSME UMR 8208 CNRS, \\ 5 bd Descartes, 77454 Marne-la-Vallée, France
}

\begin{abstract}
A fairly large family of asymptotic elastodynamic homogenization methods is shown to be derivable from Willis exact elastodynamic homogenization theory for periodic media under appropriate approximation assumptions about, for example, frequencies, wavelengths and phase contrast. In light of this result, two long-wavelength and lowfrequency asymptotic elastodynamic approaches are carefully analyzed and compared in connection with higher-order strain-gradient media. In particular, these approaches are proved to be unable to capture, at least in the one-dimensional setting, the optical branches of the dispersion curve. As an example, a two-phase string is thoroughly studied so as to illustrate the main results of the present work.
\end{abstract}

Keywords: Homogenization, elastodynamics, asymptotics, effective motion equation

\section{Introduction}

For the elastodynamic homogenization of periodically inhomogeneous media, a large family of methods based on asymptotic analysis (see, for example, Antonakakis et al. 2014; Auriault and Bonnet 1985; Auriault and Boutin 2012; Boutin and Auriault 1993; Boutin et al. 2014; Colquitt et al. 2014; Craster et al. 2010; Daya et al. 2002; Nolde et al. 2011) has been proposed and developed since the pioneer works of Bensoussan et al. (1978) and Sanchez-Palencia (1980). Making the key hypothesis of scale separation, or long wavelengths (LW), and adopting some additional assumptions such as low-frequency (LF), finite-frequency (FF), high or low phase contrast, asymptotic

\footnotetext{
*Corresponding author

Email addresses: hussein.nassar@univ-paris-est.fr (H. Nassar), qi-chang.he@univ-paris-est.fr (Q.-C. He), nicolas.auffray@univ-paris-est.fr (N. Auffray)
} 
elastodynamic homogenization approaches consist in first expanding and computing the relevant local (or microscopic) fields term by term up to, theoretically speaking, getting an arbitrarily high accuracy, and then constructing the macroscopic fields and the effective constitutive properties by carrying out appropriate volume averages over a unit cell. Even though these approaches have been proven to be useful and efficient in many situations, two important questions remain largely open. First, apart from guiding asymptotic expansions, does the scale separation hypothesis also play a role in defining the effective fields for a periodic medium? Second, what is the effective elastodynamic behavior if the asymptotic expansions are made up to infinite order?

At the same time and independently, J.R. Willis initiated and presented an elegant elastodynamic homogenization theory for periodically and randomly inhomogeneous media (Willis, 1980a,b, 1981, 1985, 1997). Recently, owing to increasing interest in acoustic metamaterials and cloaking (see, e.g., Chen and Chan 2010; Lee et al. 2012; Liu et al. 2000; Milton et al. 2006; Norris 2008; Norris and Shuvalov 2011), Willis theory has been substantially developed by him-self and other researchers (Milton and Willis, 2007, 2010; Nassar et al., 2015; Nemat-Nasser and Srivastava, 2011, 2012, 2013; Nemat-Nasser et al., 2011; Norris et al., 2012; Shuvalov et al., 2011; Srivastava and Nemat-Nasser, 2011; Willis, 2009, 2011, 2012). In contrast with asymptotic homogenization approaches, Willis theory is exact and does not make any scale separation hypothesis. In addition, the effective elastodynamic constitutive law obtained by Willis theory for a homogenized periodic medium produces the same dispersion relation as in the initial periodic one (see, e.g., Nemat-Nasser and Srivastava 2011). However, Willis effective elastodynamic behavior is in general nonlocal both in time and space, so that its numerical determination and implementation are tough and quite expensive. Further, because of the generality of Willis theory, some supplementary conditions need being specified and imposed for its application to be physically sound. This issue has been addressed and studied in our recent work about periodic media (Nassar et al., 2015).

The principal purpose of the present work is to reveal the connections between asymptotic elastodynamic homogenization approaches and Willis relevant exact theory. By achieving this objective, we hope to gain a deep insight into them and contribute to their respective development. The main results obtained by the present work can be summarized as follows:

(i) It is demonstrated that a quite large class of asymptotic elastodynamic homogenization approaches can be derived as approximations to Willis exact theory for periodic media under appropriate assumptions on loadings, microstructure and/or phase properties. Precisely, after introducing a scaling, an asymptotic theory can be deduced by expanding Willis theory over a neighborhood de- 
fined by the scaling. The resulting asymptotic theory can in turn serve as approximating and interpreting Willis theory.

(ii) Under the LW and LF hypotheses, two asymptotic elastodynamic homogenization approaches are explicitly deduced from Willis theory so that they extend to the elastodynamic context the asymptotic elastostatic ones proposed by Boutin (1996) and Smyshlyaev and Cherednichenko (2000) to account for higher-order strain-gradient effects. In addition, for highly contrasted media or under the $\mathrm{LW}$ and $\mathrm{FF}$ assumptions, the asymptotic approaches initiated by Auriault and Bonnet (1985) and by Daya et al. (2002), respectively, are shown to be consistent with a modified Willis theory first suggested by Milton and Willis (2007).

(iii) While the effective elastodynamic constitutive behavior obtained by Willis theory generates the same dispersion relation as the initial one at the microscopic level, the LW-LF asymptotic elastodynamic homogenization methods are shown to necessarily omit, at least in the one-dimensional setting, the optical branches of the dispersion curve.

(iv) For a two-phase string, Willis theory and the LW and LF asymptotic approaches are applied, and the corresponding effective elastodynamic properties are analytically obtained and numerically discussed.

The paper is organized as follows. The second section gives a short summary for Willis theory and introduces the corresponding effective displacement and body force fields which are related by an exact effective impedance incarnating the exact effective constitutive law. The third section presents asymptotic homogenization methods in two steps. The first step consists in giving two-scale representations of fields before introducing any small parameters. This demonstrates that the resulting asymptotic theories define the same notion of effective behavior as in Willis theory. In the second step, a small parameter is introduced through what is called a scaling or an imbedding, and the corresponding asymptotic expansion is carried out. In section 4 where the LF-LW hypothesis is adopted, Willis theory is used to derive two asymptotic homogenization approaches which extend to the elastodynamic setting the two ones proposed by Boutin (1996) and by Smyshlyaev and Cherednichenko (2000) in the elastostatic context. In section 5, the dispersion relations in Willis theory and asymptotic theories are carefully examined and compared. In section 6 , some of the main results obtained in the preceding sections are illustrated and discussed by studying the elastodynamic behavior of a $1 \mathrm{D}$ two-phase string. In section 7 , a short conclusion is provided. 


\section{Willis' exact elastodynamic homogenization theory: a short summary}

In the context of periodic media, the elastodynamic homogenization theory of Willis can be completely established through a purely spatial formulation in which the Bloch (or Floquet) wave expansions play a key role (Willis, 2011). Such a spatial formulation is adopted in what follows.

Consider a periodic medium $\Omega$ and let be given a pair of wavenumber $\boldsymbol{k}$ and frequency $\omega$. We prescribe over $\Omega$ a harmonic plane wave body force

$$
\boldsymbol{f}(\boldsymbol{x}, t)=\tilde{\boldsymbol{f}} e^{i(\boldsymbol{k} \cdot \boldsymbol{x}+\omega t)}
$$

where $i=\sqrt{-1}, \tilde{\boldsymbol{f}}$ is a constant force vector amplitude and $(\cdot)$ stands for the usual inner product. The periodicity hypothesis implies that the resulting displacement field $\boldsymbol{u}$ in $\Omega$ is a Bloch wave of the form

$$
\boldsymbol{u}(\boldsymbol{x}, t)=\tilde{\boldsymbol{u}}(\boldsymbol{x}) e^{i(\boldsymbol{k} \cdot \boldsymbol{x}+\omega t)}
$$

where $\tilde{\boldsymbol{u}}(\boldsymbol{x})$ is a time-independent and spatially $\mathscr{R}$-periodic displacement amplitude with $\mathscr{R}$ representing the periodicity lattice associated to $\Omega$.

In what follows, the time dependence will be dropped when there is no risk of confusion. In terms of $(\boldsymbol{u}, \boldsymbol{f})$, the harmonic motion equation over $\Omega$ can be written as

$$
\boldsymbol{\nabla} \cdot\left[\boldsymbol{C}(\boldsymbol{x}):\left(\boldsymbol{\nabla} \otimes^{s} \boldsymbol{u}(\boldsymbol{x})\right)\right]+\boldsymbol{f}(\boldsymbol{x})=-\omega^{2} \rho(\boldsymbol{x}) \boldsymbol{u}(\boldsymbol{x}) .
$$

In this equation, the fourth-order elastic stiffness tensor $\boldsymbol{C}$ and the scalar mass density $\rho$ are periodic functions of the material point position vector $\boldsymbol{x}$; the symbols $\boldsymbol{\nabla}$. and $\boldsymbol{\nabla} \otimes^{s}$ represent the divergence and symmetric gradient operators, respectively. Accounting for (2.1) and (2.2) in (2.3), we obtain the reduced but equivalent motion equation in terms of $\tilde{\boldsymbol{u}}$ and $\tilde{\boldsymbol{f}}$ :

$$
(\boldsymbol{\nabla}+i \boldsymbol{k}) \cdot\left\{\boldsymbol{C}(\boldsymbol{x}):\left[(\boldsymbol{\nabla}+i \boldsymbol{k}) \otimes^{s} \tilde{\boldsymbol{u}}(\boldsymbol{x})\right]\right\}+\tilde{\boldsymbol{f}}=-\omega^{2} \rho(\boldsymbol{x}) \tilde{\boldsymbol{u}}(\boldsymbol{x})
$$

which holds over any unit cell $T$ of the periodic medium in question and is supplemented with the appropriate periodic boundary conditions implied by the continuity of displacements and tractions. Below, the solution to equation (2.3) is noted as $\boldsymbol{u}_{\boldsymbol{k}, \omega}$ while the one to equation (2.4) is denoted by $\tilde{\boldsymbol{u}}_{\boldsymbol{k}, \omega}$.

The effective displacement field corresponds to the plane wave obtained through averaging the periodic Bloch amplitude of the microscopic one:

$$
\boldsymbol{U}_{\boldsymbol{k}, \omega}(\boldsymbol{x}) \equiv \tilde{\boldsymbol{U}}_{\boldsymbol{k}, \omega} e^{i \boldsymbol{k} \cdot \boldsymbol{x}} \equiv \frac{1}{|T|}\left(\int_{T} \tilde{\boldsymbol{u}}_{\boldsymbol{k}, \omega}\left(\boldsymbol{x}^{\prime}\right) \mathrm{d} \boldsymbol{x}^{\prime}\right) e^{i \boldsymbol{k} \cdot \boldsymbol{x}} \equiv\left\langle\tilde{\boldsymbol{u}}_{\boldsymbol{k}, \omega}\right\rangle e^{i \boldsymbol{k} \cdot \boldsymbol{x}}
$$


where $\equiv$ states equality by definition, $T$ is a unit cell, \langle\rangle is the average-over- $T$ operator and $|T|$ is the volume of $T$. It can be shown that the effective motion equation takes the form (Willis, 1997):

$$
Z_{k, \omega} \cdot \tilde{U}_{k, \omega}=\tilde{f}
$$

where $\boldsymbol{Z}_{\boldsymbol{k}, \omega}$ is the effective impedance Hermitian second order tensor. It has the following expression:

$$
\boldsymbol{Z}_{\boldsymbol{k}, \omega}=i \omega \boldsymbol{\rho}_{\boldsymbol{k}, \omega}^{e} i \omega+i \omega \boldsymbol{S}_{\boldsymbol{k}, \omega} \cdot i \boldsymbol{k}-i \boldsymbol{k} \cdot \boldsymbol{C}_{\boldsymbol{k}, \omega}^{e} \cdot i \boldsymbol{k}
$$

where $\boldsymbol{\rho}^{e}$ is the effective mass density tensor of order $2, \boldsymbol{S}$ is a tensor of order 3 combining the stress-velocity and momentum-strain coupling terms and $\boldsymbol{C}^{e}$ is the effective elastic stiffness tensor of order 4 (see AppendixA for more details). Except in simple cases, these tensors are found numerically.

The dependence of the impedance tensor $\boldsymbol{Z}_{\boldsymbol{k}, \boldsymbol{\omega}}$ on $\boldsymbol{k}$ and $\boldsymbol{\omega}$ is in general nonpolynomial and far from being trivial. The determination of $\boldsymbol{Z}_{\boldsymbol{k}, \omega}$ necessitates solving equation (2.4) for each pair $(\boldsymbol{k}, \omega)$ and for $d$ independent loadings ${ }^{1} \tilde{\boldsymbol{f}}$ in a $d$-dimensional space. It goes without saying that such task is hard and costly. Most importantly, the knowledge of $\boldsymbol{Z}_{\boldsymbol{k}, \omega}$ at distinct points $(\boldsymbol{k}, \omega)$ does not reveal the nature of the effective behavior and is rather unsatisfying. An approach which is less accurate but more uniform over (regions of) the $(\boldsymbol{k}, \omega)$-space is preferable.

The main purpose of the present paper is to discuss different asymptotic expansions, LW-LF asymptotics in particular, of the effective impedance $\boldsymbol{Z}_{\boldsymbol{k}, \omega}$. The exact effective behavior described by $\boldsymbol{Z}_{\boldsymbol{k}, \omega}$ will then be replaced by a simpler approximate asymptotic one $\boldsymbol{Z}^{\epsilon}$ truncated at some order of accuracy $R$.

\section{Asymptotic elastodynamic homogenization theories derived as approx- imations to Willis' exact one}

Asymptotic homogenization methods proceed according to an averaging scheme based on two-scale representations of fields seemingly different from the Bloch-wavebased one used in Willis theory. Next, and before introducing any asymptotic expansions, we prove that such averages coincide with definition (2.5) which implies that Willis and asymptotic theories define the same notion of effective behavior. We recast then asymptotic methods into two steps: two-scale representation and imbedding.

\footnotetext{
${ }^{1}$ Body forces will then serve as guiding the upscaling process. Afterwards, they can be set to zero in the case of investigating free waves.
} 


\subsection{Two-scale representation}

Consider the harmonic motion equation (2.3). The displacement field solution $\boldsymbol{u}_{\omega}(\boldsymbol{x})$ depends on $\boldsymbol{x}$ due to two sources of "inhomogeneities". The first one, due to $\boldsymbol{C}(\boldsymbol{x})$ and $\rho(\boldsymbol{x})$, can be referred to as microstructure. The second one, stemming from $\boldsymbol{f}(\boldsymbol{x})$, comes thus from loading. For the sake of argument, we assume that we have a closed-form expression of $\boldsymbol{u}_{\omega}(\boldsymbol{x})$ where $\boldsymbol{x}$ appears multiple times. To each appearance, we can assign one of the two mentioned inhomogeneity sources and we replace $\boldsymbol{x}$ by an auxiliary variable, $\boldsymbol{x}^{\prime}$, each time the appearance is microstructurerelated. The obtained expression, which depends on both $\boldsymbol{x}$ and $\boldsymbol{x}^{\prime}$, corresponds to a two-scale representation of the displacement field, $\boldsymbol{u}_{\omega}\left(\boldsymbol{x}, \boldsymbol{x}^{\prime}\right)$, where loading and microstructure influences have been segregated. Such representation is particularly appealing since the effective displacement can then be defined by "averaging over microstructure" according to

$$
\boldsymbol{U}_{\omega}(\boldsymbol{x})=\left\langle\boldsymbol{u}_{\omega}\left(\boldsymbol{x}, \boldsymbol{x}^{\prime}\right)\right\rangle_{\boldsymbol{x}^{\prime}}
$$

where \langle\rangle$_{\boldsymbol{x}^{\prime}}$ stands for averaging with respect to $\boldsymbol{x}^{\prime}$ over the unit cell $T$. Operator \langle\rangle$_{\boldsymbol{x}}$ is similarly defined. We stress the fact that, by construction, when $\boldsymbol{x}^{\prime}$ is replaced by $\boldsymbol{x}$, we recover $\boldsymbol{u}_{\omega}(\boldsymbol{x})=\boldsymbol{u}_{\omega}(\boldsymbol{x}, \boldsymbol{x})$ and that we expect $\boldsymbol{u}_{\omega}\left(\boldsymbol{x}, \boldsymbol{x}^{\prime}\right)$ to be $\mathscr{R}$-periodic with respect to $\boldsymbol{x}^{\prime}$ due to the periodicity of the microstructure.

Since we do not have a closed-form expression for $\boldsymbol{u}_{\omega}(\boldsymbol{x})$, the two-scale representation $\boldsymbol{u}_{\omega}\left(\boldsymbol{x}, \boldsymbol{x}^{\prime}\right)$ is defined implicitly as the solution of the equation

$$
\left(\nabla_{\boldsymbol{x}}+\nabla_{\boldsymbol{x}^{\prime}}\right) \cdot\left\{\boldsymbol{C}\left(\boldsymbol{x}^{\prime}\right):\left[\left(\nabla_{\boldsymbol{x}}+\nabla_{\boldsymbol{x}^{\prime}}\right) \otimes^{s} \boldsymbol{u}_{\omega}\left(\boldsymbol{x}, \boldsymbol{x}^{\prime}\right)\right]\right\}+\boldsymbol{f}(\boldsymbol{x})=-\omega^{2} \rho\left(\boldsymbol{x}^{\prime}\right) \boldsymbol{u}_{\omega}\left(\boldsymbol{x}, \boldsymbol{x}^{\prime}\right) .
$$

The gradient operators $\boldsymbol{\nabla}_{\boldsymbol{x}}$ and $\boldsymbol{\nabla}_{\boldsymbol{x}^{\prime}}$ act with respect to $\boldsymbol{x}$ and $\boldsymbol{x}^{\prime}$ respectively. Note that all fields are $\mathscr{R}$-periodic in $\boldsymbol{x}^{\prime}$ and that putting $\boldsymbol{x}^{\prime}=\boldsymbol{x}$ yields back the original fields and motion equation ${ }^{2}$.

Let us prescribe a plane wave body force $\boldsymbol{f}(\boldsymbol{x})=\tilde{\boldsymbol{f}} e^{i \boldsymbol{k} \cdot \boldsymbol{x}}$. The above equation having coefficients homogeneous in $\boldsymbol{x}$, its solution $\boldsymbol{u}_{\boldsymbol{k}, \omega}\left(\boldsymbol{x}, \boldsymbol{x}^{\prime}\right)$ can be looked for in the form

$$
\boldsymbol{u}_{\boldsymbol{k}, \omega}\left(\boldsymbol{x}, \boldsymbol{x}^{\prime}\right)=\tilde{\boldsymbol{u}}_{\boldsymbol{k}, \omega}\left(\boldsymbol{x}^{\prime}\right) e^{i \boldsymbol{k} \cdot \boldsymbol{x}} .
$$

The two-scale motion equation becomes

$$
\left(\nabla_{\boldsymbol{x}^{\prime}}+i \boldsymbol{k}\right) \cdot\left\{\boldsymbol{C}\left(\boldsymbol{x}^{\prime}\right):\left[\left(\boldsymbol{\nabla}_{\boldsymbol{x}^{\prime}}+i \boldsymbol{k}\right) \otimes^{s} \tilde{\boldsymbol{u}}_{\boldsymbol{k}, \omega}\left(\boldsymbol{x}^{\prime}\right)\right]\right\}+\tilde{\boldsymbol{f}}=-\omega^{2} \rho\left(\boldsymbol{x}^{\prime}\right) \tilde{\boldsymbol{u}}_{\boldsymbol{k}, \omega}\left(\boldsymbol{x}^{\prime}\right),
$$

\footnotetext{
${ }^{2}$ In particular, the following derivation rule holds:$$
\left(\nabla_{\boldsymbol{x}}+\nabla_{\boldsymbol{x}^{\prime}}\right) \otimes^{s} \boldsymbol{u}_{\omega}\left(\boldsymbol{x}, \boldsymbol{x}^{\prime}=\boldsymbol{x}\right)=\nabla_{\boldsymbol{x}} \otimes^{s} \boldsymbol{u}_{\omega}(\boldsymbol{x}) .
$$ 
and needs to be solved under $\mathscr{R}$-periodic boundary conditions and homogeneous body force amplitude $\tilde{\boldsymbol{f}}$. The foregoing equation is identical to equation (2.4) in Willis theory formulated through Bloch waves. Therefore, the displacement solutions $\boldsymbol{u}_{\boldsymbol{k}, \omega}$ are the same for both problems. Its two-scale representation is given by (3.2), where setting $\boldsymbol{x}^{\prime}=\boldsymbol{x}$ leads to the Bloch wave solution to (2.4). Hence, averaging $\boldsymbol{u}_{\boldsymbol{k}, \omega}\left(\boldsymbol{x}, \boldsymbol{x}^{\prime}\right)$ according to definition (3.1) delivers the effective displacement field defined in (2.5). Since the effective loadings and displacements are identical for both the Willis and two-scale representation approaches, so are the corresponding effective behaviors.

Having shown that the two-scale representation scheme amounts to Willis theory for periodic media, our attention can now focus on calculating $\boldsymbol{Z}_{\boldsymbol{k}, \omega}$. Scalings, that we shall introduce next, will give rise to approximations to $\boldsymbol{Z}_{\boldsymbol{k}, \omega}$ but will not change the definition of the effective behavior.

Finally, the macroscopic displacement as defined in (3.1) is sometimes thought of as a moving average of the microscopic displacement over a unit cell. Relation (2.5) defines the macroscopic displacement as the long-wavelength component of the microscopic displacement field. In fact, the moving-average interpretation is only valid in the limit $\boldsymbol{k} \rightarrow \mathbf{0}$ and only up to the first order in $\|\boldsymbol{k}\|$ while the longwavelength-component interpretation is valid as long as $\boldsymbol{k}$ is in the first Brillouin zone (Nassar et al., 2015). Both interpretations coincide in the limit $\boldsymbol{k} \rightarrow \mathbf{0}$ because the corresponding filters (respectively, a cardinal sine and a "horizontal" hyperplane) in Fourier domain are tangent at $\boldsymbol{k}=\mathbf{0}$.

\subsection{Imbedding}

In order to approximate the effective impedance $\boldsymbol{Z}_{\boldsymbol{k}, \omega}$, we will make assumptions on the order of magnitude of several of its parameters. To this end, it may be misleading to denote the effective impedance by $\boldsymbol{Z}_{\boldsymbol{k}, \omega}$ as this quantity also depends on other parameters than $\boldsymbol{k}$ and $\omega$ such as $\boldsymbol{C}(\boldsymbol{x}), \rho(\boldsymbol{x})$ and $\mathscr{R}$. Call $p$ a generic, scalar or tensorial, parameter of the homogenization problem under consideration. For the purposes of the present section, the effective impedance will be denoted by $\boldsymbol{Z}_{p}$. It is interesting to give examples of what $p$ can be: the loading frequency $\omega$ or wavenumber $\boldsymbol{k}$, the size of an inclusion in a matrix-inclusion composite, the size of a unit cell, the mean stiffness, the mean mass density or a phase contrast parameter.

The homogenization problem we are interested in is to calculate $\boldsymbol{Z}_{p=p_{f}}$ for given values $p_{f}$ of the parameters $p$. Since this problem is hard or costly to be solved through a direct calculation, we look for a "point" $p=p_{i}$ in the space of parameters for which $\boldsymbol{Z}_{p=p_{i}}$ is known or can be "easily" calculated. Next, we try to extrapolate $\boldsymbol{Z}_{p=p_{i}}$ to a neighborhood of $p_{i}$ which contains $p=p_{f}$. A natural idea is to try to apply the Taylor series expansion $\boldsymbol{Z}_{p}$ near $p=p_{i}$ and then to replace $p$ by $p_{f}$. However, 
this entails dealing with a multi-variable Taylor expansion and, in particular, trouble arises in assigning an order of accuracy to any truncated series (for example, is $\|\boldsymbol{k}\|^{2}$ infinitely small with respect to $\omega^{3}$ or the converse ?). To circumvent this difficulty, we introduce a scaling acting as a path $p=p^{\epsilon}$ such that $p(\epsilon=0)=p_{i}$ and $p(\epsilon=1)=p_{f}$. Subsequently, we define the perturbed impedance $\boldsymbol{Z}^{\epsilon}=\boldsymbol{Z}_{p=p^{\epsilon}}$ which is to be expanded about $\epsilon=0$ or $p=p_{i}$ and truncated with respect to $\epsilon$. Then by setting $\epsilon=1$ in the truncated expansion we get an approximation of $\boldsymbol{Z}_{p=p_{f}}$.

The variable $\epsilon$, through the definition of the path $p=p^{\epsilon}$ and of the expansion neighborhood about $\epsilon=0$, gives rise to all the assumptions we make on the parameters $p$. See table 1 for examples and (non-exhaustive) references.

\begin{tabular}{l|l|l} 
Expansion type & $p^{\epsilon}$ & Assumptions \\
\hline LW-LF & $\boldsymbol{k}^{\epsilon}=\epsilon \boldsymbol{k}$, & $\|\boldsymbol{k}\| \ll 2 \pi / \ell$, \\
(Boutin and Auriault, 1993) & $\omega^{\epsilon}=\epsilon \omega$ & $\omega \ll \omega_{o}$ \\
\hline LW-FF & $\boldsymbol{k}^{\epsilon}=\epsilon \boldsymbol{k}$, & $\|\boldsymbol{k}\| \ll 2 \pi / \ell$, \\
(Daya et al., 2002) & $\omega^{\epsilon}=\omega_{0}+\epsilon \omega_{1}+\ldots$ & $\omega \approx \omega_{0} \neq 0$ \\
\hline High-contrast (LW-LF) & $\boldsymbol{k}^{\epsilon}=\epsilon \boldsymbol{k}$, & $\|\boldsymbol{k}\| \ll 2 \pi / \ell$, \\
(Auriault and Bonnet, 1985) & $\omega^{\epsilon}=\epsilon \omega$, & $\omega \ll \omega_{o}$, \\
& $\boldsymbol{C}^{\epsilon}=\boldsymbol{C}^{2}+\chi^{1}\left(\epsilon^{2} \boldsymbol{C}^{1}-\boldsymbol{C}^{2}\right)$ & $\left\|\boldsymbol{C}^{1}\right\| \ll\left\|\boldsymbol{C}^{2}\right\|$ \\
\hline Low-contrast & $\boldsymbol{C}^{\epsilon}=\langle\boldsymbol{C}\rangle+\epsilon \boldsymbol{C}^{\prime}$, & $\left\|\boldsymbol{C}^{\prime}\right\| \ll\|\langle\boldsymbol{C}\rangle\|$, \\
(Willis, 1985, 1997) & $\rho^{\epsilon}=\langle\rho\rangle+\epsilon \rho^{\prime}$ & $\rho^{\prime} \ll\langle\rho\rangle$
\end{tabular}

Table 1: Examples of expansions from the literature. Symbol $\approx$ means "of the same order of magnitude", symbol « means "very small with respect to", $\ell$ and $\omega_{o}$ are, respectively, a characteristic length and frequency of the microstructure, $C^{1}$ and $C^{2}$ are the stiffness tensors of a two-phase composite, $\chi^{1}$ is the indicator function of phase 1 in that composite, $\boldsymbol{C}^{\prime}=\boldsymbol{C}-\langle\boldsymbol{C}\rangle, \rho^{\prime}=\rho-\langle\rho\rangle$ and $\|\boldsymbol{C}\|$ is the norm of $\boldsymbol{C}$.

Now for a chosen scaling $p^{\epsilon}$, we need to first define and then calculate $\boldsymbol{Z}^{\epsilon}$ without referring to the unknown impedance $\boldsymbol{Z}_{p}$. Recalling that the determination of $\boldsymbol{Z}_{p}$ requires solving equation (2.4), the calculation of the expansion of $\boldsymbol{Z}^{\epsilon}$ necessitates solving the perturbed motion equation deduced from (2.4) according to $p \mapsto p^{\epsilon}$. Borrowing the words of Bensoussan et al. (1978), this amounts to "imbedding" equation (2.4) into "a family of problems (parametrized by $\epsilon$ )":

$$
\left(\boldsymbol{\nabla}+i \boldsymbol{k}^{\epsilon}\right) \cdot\left\{\boldsymbol{C}^{\epsilon}(\boldsymbol{x}):\left[\left(\boldsymbol{\nabla}+i \boldsymbol{k}^{\epsilon}\right) \otimes^{s} \tilde{\boldsymbol{u}}^{\epsilon}(\boldsymbol{x})\right]\right\}+\tilde{\boldsymbol{f}}=-\left(\omega^{\epsilon}\right)^{2} \rho^{\epsilon}(\boldsymbol{x}) \tilde{\boldsymbol{u}}^{\epsilon}(\boldsymbol{x}) .
$$

While the scaling of $p=\{\boldsymbol{C}, \rho, \boldsymbol{k}, \omega\}$ can be chosen arbitrarily, scaling, or expansion, 
of the solution $\tilde{\boldsymbol{u}}$ is to be deduced so as to fulfill the above equation. Body force scaling is of no importance since $\tilde{\boldsymbol{f}}$ and $\tilde{\boldsymbol{u}}$ are in a linear relationship.

Injecting a Taylor expansion of the perturbed displacement field

$$
\tilde{\boldsymbol{u}}^{\epsilon}=\sum_{r} \tilde{\boldsymbol{u}}^{r} \epsilon^{r}
$$

into (3.3) yields a hierarchy of microscopic motion equations, one for each order in $\epsilon$. Solving these equations for $\tilde{\boldsymbol{u}}^{r}$, we obtain a hierarchy of effective motion equations in the coefficients $\tilde{\boldsymbol{U}}^{r}$ of the Taylor expansion of the perturbed effective displacement given by

$$
\tilde{\boldsymbol{U}}^{\epsilon} \equiv\left\langle\tilde{\boldsymbol{u}}^{\epsilon}\right\rangle \equiv \sum_{r} \tilde{\boldsymbol{U}}^{r} \epsilon^{r} \equiv \sum_{r}\left\langle\tilde{\boldsymbol{u}}^{r}\right\rangle \epsilon^{r} .
$$

Said effective hierarchy defines the terms $\boldsymbol{Z}^{r}$ in the expansion of $\boldsymbol{Z}^{\epsilon}$ :

$$
\boldsymbol{Z}^{\epsilon}=\sum_{r} \boldsymbol{Z}^{r} \epsilon^{r} .
$$

The last step in writing the approximate effective behavior consists in determining the approximate effective impedance of order $R$. In other words, having the scaled effective motion equation

$$
\boldsymbol{Z}^{\epsilon} \cdot \tilde{\boldsymbol{U}}^{\epsilon}=\tilde{\boldsymbol{f}},
$$

we need to define a truncated effective impedance in some sense. Two general systematic approaches have been proposed in the literature. For clarity, it is preferable to present these approaches in their original context of LW-LF asymptotics.

\section{Links between exact and LW-LF asymptotic approaches}

Effective impedances $\boldsymbol{Z}$, whether exact or approximate, depend on the Fourier variables $\boldsymbol{k}$ and $\omega$. Therefore, back in the real space-time domain, $\boldsymbol{Z}$ corresponds to an integro-differential operator which acts on the effective displacement field $\boldsymbol{U}(\boldsymbol{x}, t)$ and yields $\boldsymbol{f}(\boldsymbol{x}, t)$. The nature of this operator is determined by the way in which $\boldsymbol{Z}$ depends on $\boldsymbol{k}$ and $\omega$. LW-LF asymptotics give birth to an approximate effective impedance which is a polynomial in $\boldsymbol{k}$ and $\omega$. Accordingly, the LW-LF approximate effective behavior is local in both space and time and corresponds, to the lowest order, to the usual wave equation as will be seen. Other scalings yield other types of approximate effective behaviors, the nature of which (whether local or not for instance) depends strongly on the underlying microstructure and the expansion neighborhood.

Rather than pursuing our general discussion of asymptotic methods we will restrict our attention to selected topics in LW-LF asymptotics and make a few links with Willis exact theory through the use of Bloch wave expansions. High-contrast and FF asymptotics are briefly discussed in subsection 5.5. 


\subsection{Two scalings}

In the classical formulation of asymptotic homogenization as presented by SanchezPalencia (1980) for instance, the small parameter $\epsilon$ is introduced in the arguments of $\boldsymbol{C}$ and $\rho$ and is used to make them vary on an infinitely (as $\epsilon \rightarrow 0$ ) finer scale than the loading. The corresponding two-scale motion equation takes the form

$$
\left(\nabla_{\boldsymbol{x}}+\nabla_{\boldsymbol{x}^{\prime}}\right) \cdot\left\{\boldsymbol{C}\left(\boldsymbol{x}^{\prime} / \epsilon\right):\left[\left(\nabla_{\boldsymbol{x}}+\nabla_{\boldsymbol{x}^{\prime}}\right) \otimes^{s} \boldsymbol{u}^{\epsilon}\left(\boldsymbol{x}, \boldsymbol{x}^{\prime}\right)\right]\right\}+\boldsymbol{f}(\boldsymbol{x})=-\omega^{2} \rho\left(\boldsymbol{x}^{\prime} / \epsilon\right) \boldsymbol{u}^{\epsilon}\left(\boldsymbol{x}, \boldsymbol{x}^{\prime}\right) .
$$

The Fourier transform of the above equation with respect to $\boldsymbol{x}$ results in

$$
\left(i \boldsymbol{k}+\nabla_{\boldsymbol{x}^{\prime}}\right) \cdot\left\{\boldsymbol{C}\left(\boldsymbol{x}^{\prime} / \epsilon\right):\left[\left(i \boldsymbol{k}+\nabla_{\boldsymbol{x}^{\prime}}\right) \otimes^{s} \tilde{\boldsymbol{u}}^{\epsilon}\left(\boldsymbol{x}^{\prime}\right)\right]\right\}+\tilde{\boldsymbol{f}}=-\omega^{2} \rho\left(\boldsymbol{x}^{\prime} / \epsilon\right) \tilde{\boldsymbol{u}}^{\epsilon}\left(\boldsymbol{x}^{\prime}\right),
$$

which is a particular case of the general equation (3.3) with $\boldsymbol{C}^{\epsilon}\left(\boldsymbol{x}^{\prime}\right)=\boldsymbol{C}\left(\boldsymbol{x}^{\prime} / \epsilon\right)$ and $\rho^{\epsilon}\left(\boldsymbol{x}^{\prime}\right)=\rho\left(\boldsymbol{x}^{\prime} / \epsilon\right)$. Upon the change of variable $\boldsymbol{y}=\boldsymbol{x}^{\prime} / \epsilon$, the above equation becomes

$$
\left(i \boldsymbol{k}+\frac{1}{\epsilon} \nabla_{\boldsymbol{y}}\right) \cdot\left\{\boldsymbol{C}(\boldsymbol{y}):\left[\left(i \boldsymbol{k}+\frac{1}{\epsilon} \nabla_{\boldsymbol{y}}\right) \otimes^{s} \hat{\boldsymbol{u}}^{\epsilon}(\boldsymbol{y})\right]\right\}+\tilde{\boldsymbol{f}}=-\omega^{2} \rho(\boldsymbol{y}) \hat{\boldsymbol{u}}^{\epsilon}(\boldsymbol{y})
$$

with $\hat{\boldsymbol{u}}^{\epsilon}(\boldsymbol{y})=\tilde{\boldsymbol{u}}^{\epsilon}\left(\boldsymbol{x}^{\prime}\right)$. Rearranging the powers of $\epsilon$, we get:

$$
\left(\nabla_{\boldsymbol{y}}+i \epsilon \boldsymbol{k}\right) \cdot\left\{\boldsymbol{C}(\boldsymbol{y}):\left[\left(\nabla_{\boldsymbol{y}}+i \epsilon \boldsymbol{k}\right) \otimes^{s}\left(\frac{1}{\epsilon^{2}} \hat{\boldsymbol{u}}^{\epsilon}(\boldsymbol{y})\right)\right]\right\}+\tilde{\boldsymbol{f}}=-(\epsilon \omega)^{2} \rho(\boldsymbol{y})\left(\frac{1}{\epsilon^{2}} \hat{\boldsymbol{u}}^{\epsilon}(\boldsymbol{y})\right)
$$

which is another particular case of (3.3) and where $\boldsymbol{y}$ becomes a dummy variable and needs not to be specified.

The above two scalings are therefore related to one another in the following way:

$$
\tilde{\boldsymbol{u}}_{C^{\epsilon}, \rho^{\epsilon}}\left(\boldsymbol{x}^{\prime}\right)=\epsilon^{2} \tilde{\boldsymbol{u}}_{\epsilon \boldsymbol{k}, \epsilon \omega}(\boldsymbol{y}),
$$

with $\boldsymbol{y}=\boldsymbol{x}^{\prime} / \epsilon$. Hence, reducing the unit cell size by a factor of $\epsilon$ is equivalent to reducing the wavelength and the frequency of the loading by the same factor while rescaling displacements by a factor of $\epsilon^{2}$. This relation shows that the classical approach, based on $\boldsymbol{C}^{\epsilon}$ and $\rho^{\epsilon}$, is equivalent to the one based on LW-LF asymptotics. Averaging (4.2), we see that the perturbed effective impedance can also be recast in two ways:

$$
\boldsymbol{Z}^{\epsilon}=\boldsymbol{Z}_{\boldsymbol{C}^{\epsilon}, \rho^{\epsilon}}=\frac{1}{\epsilon^{2}} \boldsymbol{Z}_{\epsilon \boldsymbol{k}, \epsilon \omega}
$$

The above equivalency relation between the classical scaling $\left\{\boldsymbol{C}^{\epsilon}, \rho^{\epsilon}\right\}$ and the LW-LF one $\{\epsilon \boldsymbol{k}, \epsilon \omega\}$ shows again how the asymptotic homogenization theory can be derived from Willis theory. More details will be given subsequently. 
The perturbed full Bloch wave $\boldsymbol{u}^{\epsilon}(\boldsymbol{x})=\tilde{\boldsymbol{u}}^{\epsilon}(\boldsymbol{x}) e^{i \boldsymbol{k} \cdot \boldsymbol{x}}$ response to the plane wave loading $\boldsymbol{f}(\boldsymbol{x})=\tilde{\boldsymbol{f}} e^{i \boldsymbol{k} \cdot \boldsymbol{x}}$ now admits the two-scale representation:

$$
\boldsymbol{u}^{\epsilon}\left(\boldsymbol{x}, \boldsymbol{x}^{\prime}\right)=\tilde{\boldsymbol{u}}^{\epsilon}\left(\boldsymbol{x}^{\prime}\right) e^{i \boldsymbol{k} \cdot \boldsymbol{x}}=\epsilon^{2} \tilde{\boldsymbol{u}}_{\epsilon \boldsymbol{k}, \epsilon \omega}(\boldsymbol{y}) e^{i \boldsymbol{k} \cdot \boldsymbol{x}}=\epsilon^{2} \tilde{\boldsymbol{u}}_{\epsilon \boldsymbol{k}, \epsilon \omega}\left(\boldsymbol{x}^{\prime} / \epsilon\right) e^{i \boldsymbol{k} \cdot \boldsymbol{x}}
$$

The one-variable representation of the perturbed solution can be recovered by mapping $\boldsymbol{x}^{\prime} \mapsto \boldsymbol{x}$ :

$$
\boldsymbol{u}^{\epsilon}(\boldsymbol{x})=\epsilon^{2} \tilde{\boldsymbol{u}}_{\epsilon \boldsymbol{k}, \epsilon \omega}(\boldsymbol{x} / \epsilon) e^{i \boldsymbol{k} \cdot \boldsymbol{x}} .
$$

Bloch waves appear then to naturally separate the slow, or macroscopic, variable $\boldsymbol{k} \cdot \boldsymbol{x}$ from the fast, or microscopic, variable $\boldsymbol{x} / \epsilon$. As expected, for $\epsilon=1$, we recover the unperturbed displacement field.

The field $\tilde{\boldsymbol{u}}_{\epsilon \boldsymbol{k}, \epsilon \omega}(\boldsymbol{x} / \epsilon)$ is $\epsilon \mathscr{R}$-periodic with respect to $\boldsymbol{x}$ and admits a Fourier series expansion over the reciprocal lattice $\epsilon^{-1} \mathscr{R}^{*}=\left\{\epsilon^{-1} \boldsymbol{\xi}, \boldsymbol{\xi} \in \mathscr{R}^{*}\right\}$ where $\mathscr{R}^{*}$ is the reciprocal lattice of $\mathscr{R}$. It can then be seen as a function of the fast variables $\epsilon^{-1} \boldsymbol{\xi} \cdot \boldsymbol{x}$ whereas the slow variable is $\boldsymbol{k} \cdot \boldsymbol{x}$. The parameter $\epsilon$ then plays the role of comparing the magnitudes of $\boldsymbol{k}$ and $\boldsymbol{\xi} \in \mathscr{R}^{*}$. The requirement of scale separation, allowing for a real, rather than conventional, distinction between fast and slow variables, can be formulated as

$$
\|\boldsymbol{k}\| \ll\|\boldsymbol{\xi}\|, \quad \forall \boldsymbol{\xi} \in \mathscr{R}^{*}-\{\mathbf{0}\},
$$

the smallest non-null $\|\boldsymbol{\xi}\|$ being comparable to the inverse of the unit cell characteristic length.

\subsection{Hierarchical motion equations}

Following the discussion at the end of section 3.2, we write the first three motion equations of orders $\epsilon^{-2}, \epsilon^{-1}$ and $\epsilon^{0}=1$ obtained by applying the Taylor expansion to $\hat{\boldsymbol{u}}^{\epsilon}$ in equation (4.1) about $\epsilon=0$. In fact, this has been done with great details ${ }^{3}$ by Boutin and Auriault (1993) and is recalled here for use in the following sections. Noting that $\hat{\boldsymbol{u}}$ corresponds to $\tilde{\boldsymbol{u}}$ with a rescaled space variable, the lowest-order motion equation reads

$$
\boldsymbol{\nabla} \cdot\left[\boldsymbol{C}:\left(\boldsymbol{\nabla} \otimes^{s} \hat{\boldsymbol{u}}_{\boldsymbol{k}, \omega}^{0}\right)\right]=\mathbf{0}
$$

which yields

$$
\hat{\boldsymbol{u}}_{\boldsymbol{k}, \omega}^{0}(\boldsymbol{y})=\tilde{\boldsymbol{U}}_{\boldsymbol{k}, \omega}^{0}
$$

The second one takes the form

$$
\boldsymbol{\nabla} \cdot\left[\boldsymbol{C}:\left(\boldsymbol{\nabla} \otimes{ }^{s} \hat{\boldsymbol{u}}_{\boldsymbol{k}, \omega}^{1}+i \boldsymbol{k} \otimes^{s} \tilde{\boldsymbol{U}}_{\boldsymbol{k}, \omega}^{0}\right)\right]=\mathbf{0}
$$

\footnotetext{
${ }^{3}$ although with no body forces.
} 
which leads to

$$
\hat{\boldsymbol{u}}_{\boldsymbol{k}, \omega}^{1}(\boldsymbol{y})=\tilde{\boldsymbol{U}}_{\boldsymbol{k}, \omega}^{1}+\boldsymbol{A}(\boldsymbol{y}):\left(i \boldsymbol{k} \otimes{ }^{s} \tilde{\boldsymbol{U}}_{\boldsymbol{k}, \omega}^{0}\right),
$$

where $\boldsymbol{A}$ is a microstructure dependent, $(\boldsymbol{k}, \omega)$-independent, zero-average localization operator. Finally, the third one, averaged with respect to $\boldsymbol{y} \in T$, gives the classical quasistatic homogenized motion equation:

$$
i \boldsymbol{k} \cdot\left[\langle\boldsymbol{C}+\boldsymbol{C}: \nabla \boldsymbol{A}\rangle:\left(i \boldsymbol{k} \otimes{ }^{s} \tilde{\boldsymbol{U}}_{\boldsymbol{k}, \omega}^{0}\right)\right]+\tilde{\boldsymbol{f}}=-\omega^{2}\langle\rho\rangle \tilde{\boldsymbol{U}}_{\boldsymbol{k}, \omega}^{0} .
$$

Therefore, the lowest order term $\boldsymbol{Z}_{\boldsymbol{k}, \boldsymbol{\omega}}^{0}$ in the LW-LF Taylor expansion of $\boldsymbol{Z}$ is equal to

$$
\boldsymbol{Z}_{\boldsymbol{k}, \omega}^{0}=\boldsymbol{k} \cdot\left\langle\boldsymbol{C}+\boldsymbol{C}:\left(\boldsymbol{\nabla} \otimes^{s} \boldsymbol{A}\right)\right\rangle \cdot \boldsymbol{k}-\omega^{2}\langle\rho\rangle \boldsymbol{I},
$$

where $\boldsymbol{I}$ is the second order identity tensor. Note that using Bloch waves to obtain this classical result of homogenization theory is not new (see, for instance, Turbé, 1982, 1989). Here, we show that Bloch waves can similarly serve as establishing a systematic connection with Willis theory.

Writing and solving higher-order motion equations allow to calculate all other terms $\boldsymbol{Z}^{r}$. Next, we present two methods of defining the approximate effective impedance of a given order $R$ denoted by $\boldsymbol{Z}^{(R)}$ for the first method and by $\boldsymbol{Z}^{[R]}$ for the second one.

\subsection{Approximate effective behavior: momentum balance-based approach}

This first approach is an elastodynamic extension of the method used by Boutin (1996) to describe microstructural effects in elastostatics.

Having calculated the expansion (3.6) up to order $R$, the approximate effective behavior can be defined through the approximate effective impedance

$$
\boldsymbol{Z}_{\boldsymbol{k}, \omega}^{(R)}=\sum_{r \leq R} \boldsymbol{Z}_{\boldsymbol{k}, \omega}^{r}
$$

The approximate effective motion equation accordingly reads

$$
Z_{\boldsymbol{k}, \omega}^{(R)} \cdot \tilde{\boldsymbol{U}}_{\boldsymbol{k}, \omega}=\tilde{\boldsymbol{f}}
$$

The lowest order approximate effective behavior corresponds therefore to $\boldsymbol{Z}^{(0)}=\boldsymbol{Z}^{0}$ given in equation (4.4).

Let us now specify the connection between Willis exact theory and the present LW-LF asymptotic theory. Thanks to equations (4.3) and (2.7), we have

$$
\boldsymbol{Z}^{\epsilon}=i \omega \boldsymbol{\rho}_{\epsilon \boldsymbol{k}, \epsilon \omega}^{e} i \omega+i \omega \boldsymbol{S}_{\epsilon \boldsymbol{k}, \epsilon \omega} \cdot i \boldsymbol{k}-i \boldsymbol{k} \cdot \boldsymbol{C}_{\epsilon \boldsymbol{k}, \epsilon \omega}^{e} \cdot i \boldsymbol{k}
$$


where an $\epsilon^{2}$ factor is canceled. Indeed, a general formula giving the expansion of $\boldsymbol{Z}^{\epsilon}$ is

$$
\begin{aligned}
\boldsymbol{Z}^{\epsilon}=\sum_{r} \epsilon^{r} \sum_{\alpha+\beta=r} \frac{(i \omega)^{\beta}}{\alpha ! \beta !} \frac{\partial^{r}}{\partial\left(i \boldsymbol{k}^{\prime}\right)^{\alpha} \partial\left(i \omega^{\prime}\right)^{\beta}}\left[i \omega \boldsymbol{\rho}_{\boldsymbol{k}^{\prime}, \omega^{\prime}}^{e} i \omega\right. \\
\left.\quad+i \omega \boldsymbol{S}_{\boldsymbol{k}^{\prime}, \omega^{\prime}} \cdot i \boldsymbol{k}-i \boldsymbol{k} \cdot \boldsymbol{C}_{\boldsymbol{k}^{\prime}, \omega^{\prime}}^{e} \cdot i \boldsymbol{k}\right]\left.\right|_{\boldsymbol{k}^{\prime}=0, \omega^{\prime}=0} \odot^{\alpha}\left[\otimes^{\alpha} i \boldsymbol{k}\right]
\end{aligned}
$$

where $\odot^{\alpha}$ stands for $\alpha$ times contraction and $\otimes^{\alpha} i \boldsymbol{k}$ represents the $\alpha^{\text {th }}$ tensor power of $i \boldsymbol{k}$. Thus, we have

$$
\begin{aligned}
\boldsymbol{Z}_{\boldsymbol{k}, \omega}^{(R)}=\sum_{\alpha+\beta \leq R} \frac{(i \omega)^{\beta}}{\alpha ! \beta !} \frac{\partial^{\alpha+\beta}}{\partial\left(i \boldsymbol{k}^{\prime}\right)^{\alpha} \partial\left(i \omega^{\prime}\right)^{\beta}}\left[i \omega \boldsymbol{\rho}_{\boldsymbol{k}^{\prime}, \omega^{\prime}}^{e} i \omega\right. \\
\\
\left.\quad+i \omega \boldsymbol{S}_{\boldsymbol{k}^{\prime}, \omega^{\prime}} \cdot i \boldsymbol{k}-i \boldsymbol{k} \cdot \boldsymbol{C}_{\boldsymbol{k}^{\prime}, \omega^{\prime}}^{e} \cdot i \boldsymbol{k}\right]\left.\right|_{\boldsymbol{k}^{\prime}=0, \omega^{\prime}=0} \odot^{\alpha}\left[\otimes^{\alpha} i \boldsymbol{k}\right] .
\end{aligned}
$$

In the above expansions, we can restrict the summation to even powers of $\omega$ since all motion equations depend on $\omega$ uniquely through $\omega^{2}$ (this would not be the case for a linear visco-elastic behavior for example). In particular, for $R=0$, the above sum reduces to the sole term

$$
\boldsymbol{Z}_{\boldsymbol{k}, \omega}^{(0)}=\boldsymbol{Z}_{\boldsymbol{k}, \omega}^{0}=i \omega \boldsymbol{\rho}_{\mathbf{0}, 0}^{e} i \omega+i \omega \boldsymbol{S}_{\mathbf{0}, 0} \cdot i \boldsymbol{k}-i \boldsymbol{k} \cdot \boldsymbol{C}_{\mathbf{0}, 0}^{e} \cdot i \boldsymbol{k},
$$

meaning that the Willis effective constitutive parameters, to the lowest order, thanks to equation (4.4), are given by:

$$
\begin{aligned}
\boldsymbol{\rho}_{\mathbf{0}, 0}^{e} & =\langle\rho\rangle \boldsymbol{I}, \\
\boldsymbol{S}_{\mathbf{0}, 0} & =\mathbf{0}, \\
\boldsymbol{C}_{\mathbf{0}, 0}^{e} & =\left\langle\boldsymbol{C}+\boldsymbol{C}: \boldsymbol{\nabla} \otimes^{s} \boldsymbol{A}\right\rangle .
\end{aligned}
$$

The expression of $\boldsymbol{Z}^{(R)}$ for $R \geq 1$ requires pushing further in the hierarchical motion equations as noted earlier and will allow to characterize the higher order terms in the expansion of the Willis effective constitutive parameters. Finally, to obtain the corresponding approximate effective motion equation in the real space-time domain, we simply use the mappings $i \omega \mapsto \partial / \partial t$ and $i \boldsymbol{k} \mapsto \boldsymbol{\nabla}$ in the expression of $\boldsymbol{Z}^{(R)}$. For $R=2$, the approximate effective behavior is that of a strain-gradient medium with micro-inertia (i.e., new terms with time derivatives) whose motion equation involves the following derivatives of $\boldsymbol{U}$ :

$$
\frac{\partial^{\alpha+2 \beta}}{\partial \boldsymbol{x}^{\alpha} \partial t^{2 \beta}} \boldsymbol{U} \text { for } \alpha+2 \beta \leq 4
$$

An explicit example of such approximate effective motion equation is given in section 6 . 


\subsection{Approximate effective behavior: energy-based approach}

This second approach is based on two ingredients: a localization operator and a variational formulation. This approach, introduced by Smyshlyaev and Cherednichenko (2000) for elastostatics, is generalized here to elastodynamics.

As for the localization operator, one can summarize the results of the first two motion equations of the hierarchy by writing

$$
\begin{aligned}
\hat{\boldsymbol{u}}^{\epsilon}(\boldsymbol{y}) & =\hat{\boldsymbol{u}}_{\boldsymbol{k}, \omega}^{0}(\boldsymbol{y})+\epsilon \hat{\boldsymbol{u}}_{\boldsymbol{k}, \omega}^{1}(\boldsymbol{y})+O\left(\epsilon^{2}\right) \\
& =\tilde{\boldsymbol{U}}_{\boldsymbol{k}, \omega}^{0}+\epsilon \tilde{\boldsymbol{U}}_{\boldsymbol{k}, \omega}^{1}+\epsilon \boldsymbol{A}(\boldsymbol{y}): i \boldsymbol{k} \otimes^{s} \tilde{\boldsymbol{U}}_{\boldsymbol{k}, \omega}^{0}+O\left(\epsilon^{2}\right) \\
& =\tilde{\boldsymbol{U}}^{\epsilon}+\epsilon \boldsymbol{A}(\boldsymbol{y}): i \boldsymbol{k} \otimes^{s} \tilde{\boldsymbol{U}}^{\epsilon}+O\left(\epsilon^{2}\right),
\end{aligned}
$$

where $\boldsymbol{A}$ appears as the first order term of some localization operator $\boldsymbol{L}^{\epsilon}$ defined by:

$$
\hat{\boldsymbol{u}}^{\epsilon}(\boldsymbol{y})=\boldsymbol{L}^{\epsilon}(\boldsymbol{y}) \cdot \tilde{\boldsymbol{U}}^{\epsilon} .
$$

As for the variational formulation, starting from (4.1), it can be seen that $\hat{\boldsymbol{u}}^{\epsilon}$ is the stationary point of the action defined by

$$
\begin{aligned}
& \frac{1}{2}\left\langle\left[\left(\frac{1}{\epsilon} \boldsymbol{\nabla}+i \boldsymbol{k}\right) \otimes^{s} \hat{\boldsymbol{u}}^{\epsilon}\right]^{* T}: \boldsymbol{C}:\left[\left(\frac{1}{\epsilon} \boldsymbol{\nabla}+i \boldsymbol{k}\right) \otimes^{s} \hat{\boldsymbol{u}}^{\epsilon}\right]-\omega^{2} \rho \hat{\boldsymbol{u}}^{\epsilon * T} \cdot \hat{\boldsymbol{u}}^{\epsilon}\right\rangle-\Re\left(\left\langle\hat{\boldsymbol{u}}^{\epsilon *} \cdot \tilde{\boldsymbol{f}}\right\rangle\right) \\
= & \frac{1}{2}\left\langle\left[\left(\frac{1}{\epsilon} \boldsymbol{\nabla}+i \boldsymbol{k}\right) \otimes^{s} \hat{\boldsymbol{u}}^{\epsilon}\right]^{* \top}: \boldsymbol{C}:\left[\left(\frac{1}{\epsilon} \boldsymbol{\nabla}+i \boldsymbol{k}\right) \otimes^{s} \hat{\boldsymbol{u}}^{\epsilon}\right]-\omega^{2} \rho \hat{\boldsymbol{u}}^{\epsilon * \top} \cdot \hat{\boldsymbol{u}}^{\epsilon}\right\rangle-\Re\left(\tilde{\boldsymbol{U}}^{\epsilon *} \cdot \tilde{\boldsymbol{f}}\right),
\end{aligned}
$$

over the set of admissible displacement fields ( $\mathscr{R}$-periodic fields basically), where $\left(^{\top}\right)$ stands for transposition and $\Re$ symbolizes the real part of a complex number. However, we know that such stationary point can be written as in equation (4.10). Upon the corresponding change of unknown field, the action becomes

$$
\begin{aligned}
\frac{1}{2} \tilde{\boldsymbol{U}}^{\epsilon *} \cdot\left\langle\left[\left(\frac{1}{\epsilon} \boldsymbol{\nabla}+i \boldsymbol{k}\right) \otimes^{s} \boldsymbol{L}^{\epsilon}\right]^{* \top}\right. & \left.: \boldsymbol{C}:\left[\left(\frac{1}{\epsilon} \boldsymbol{\nabla}+i \boldsymbol{k}\right) \otimes^{s} \boldsymbol{L}^{\epsilon}\right]-\omega^{2} \rho \boldsymbol{L}^{\epsilon * \top} \cdot \boldsymbol{L}^{\epsilon}\right\rangle \cdot \tilde{\boldsymbol{U}}^{\epsilon} \\
& -\Re\left(\tilde{\boldsymbol{U}}^{\epsilon *} \cdot \tilde{\boldsymbol{f}}\right)=\frac{1}{2} \tilde{\boldsymbol{U}}^{\epsilon *} \cdot \boldsymbol{Z}^{\epsilon} \cdot \tilde{\boldsymbol{U}}^{\epsilon}-\Re\left(\tilde{\boldsymbol{U}}^{\epsilon *} \cdot \tilde{\boldsymbol{f}}\right) .
\end{aligned}
$$

Finally, instead of truncating the expansion of $\boldsymbol{Z}^{\epsilon}$, the one of $\boldsymbol{L}^{\epsilon}$ is truncated at order $R$ to yield an approximate localization operator $\boldsymbol{L}^{(R)}$ which is then injected into (4.11). To sum up, we define the approximate effective impedance by

$$
\boldsymbol{Z}_{\boldsymbol{k}, \omega}^{[2 R]}=\left\langle\left[(\boldsymbol{\nabla}+i \boldsymbol{k}) \otimes^{s} \boldsymbol{L}_{\boldsymbol{k}, \omega}^{(R)}\right]^{* \top}: \boldsymbol{C}:\left[(\boldsymbol{\nabla}+i \boldsymbol{k}) \otimes^{s} \boldsymbol{L}_{\boldsymbol{k}, \omega}^{(R)}\right]-\omega^{2} \rho \boldsymbol{L}_{\boldsymbol{k}, \omega}^{(R) * \mathrm{~T}} \cdot \boldsymbol{L}_{\boldsymbol{k}, \omega}^{(R)}\right\rangle .
$$

The approximate effective motion equation is accordingly

$$
Z_{k, \omega}^{[2 R]} \cdot \tilde{\boldsymbol{U}}_{k, \omega}=\tilde{\boldsymbol{f}}
$$


Impedance $\boldsymbol{Z}^{[2 R]}$ is not a partial sum of any Taylor series and is different from $\boldsymbol{Z}^{(2 R)}$. In particular, $\boldsymbol{Z}^{[0]}$ is different from the first term in the expansion $\boldsymbol{Z}^{0}$. In fact, since $\boldsymbol{L}^{(0)}=\boldsymbol{I}$ as can be seen from equation (4.9), we have:

$$
\boldsymbol{Z}_{\boldsymbol{k}, \omega}^{[0]}=\boldsymbol{k} \cdot\langle\boldsymbol{C}\rangle \cdot \boldsymbol{k}-\omega^{2}\langle\rho\rangle \boldsymbol{I}
$$

Using again equation (4.9), we see that for $R=1$, the approximate effective behavior is also that of a strain-gradient medium with micro-inertia whose motion equation involves the same derivatives as in (4.8) except for $\partial^{4} \boldsymbol{U} / \partial t^{4}$. An explicit example of such approximate effective motion equation is given in section 6 .

\subsection{Discussion}

It is of interest to discuss some of the differences distinguishing the above two approaches. Comparing, in particular, $\boldsymbol{Z}^{(2)}$ and $\boldsymbol{Z}^{[2]}$ will be enough.

First, both approximate impedances yield a medium of order 4 in the sense that the highest order derivative of $\boldsymbol{U}$ appearing in the motion equation is of order 4 . However, $\boldsymbol{Z}^{(2)}$ involves a $4^{\text {th }}$-order time derivative while $\boldsymbol{Z}^{[2]}$ does not and is hence formally identical to the impedance of the strain-gradient medium derived by Mindlin (1964).

Second, the most important advantage of the energy-based approach is that $\boldsymbol{Z}^{[2]}$ guarantees a positive definite elastic energy. As a matter of fact, truncating $\boldsymbol{L}^{\epsilon}$ according to (4.9) amounts to changing the set of admissible fields of the action (4.11) and not the action itself. Since $\boldsymbol{C}$ is positive definite, so is the elastic energy defined by $\boldsymbol{Z}^{[2]}$. The momentum balance-based approach on the other hand does not necessarily yield a positive definite elastic energy. However, if one were to interpret the motion equation (4.5) asymptotically and solve for the $\tilde{\boldsymbol{U}}^{r}$ instead of $\tilde{\boldsymbol{U}}$, then equation (4.5) will be replaced by the following hierarchy:

$$
\begin{aligned}
Z_{\boldsymbol{k}, \omega}^{0} \cdot \tilde{\boldsymbol{U}}_{\boldsymbol{k}, \omega}^{0} & =\tilde{\boldsymbol{f}} \\
Z_{\boldsymbol{k}, \omega}^{0} \cdot \tilde{\boldsymbol{U}}_{\boldsymbol{k}, \omega}^{1}+\boldsymbol{Z}_{\boldsymbol{k}, \omega}^{1} \cdot \tilde{\boldsymbol{U}}_{\boldsymbol{k}, \omega}^{0} & =\mathbf{0} \ldots
\end{aligned}
$$

The elastic energy at each stage remains therefore positive definite since $\boldsymbol{Z}^{0}$ defines a positive definite effective elastic stiffness tensor as is well-known.

Third, only the momentum balance-based approach defines a "good" limit process in the sense that $\boldsymbol{Z}^{(0)}$ is equal to the correct homogenization limit while $\boldsymbol{Z}^{[0]}$ is some kind of a low-contrast approximation of said limit. At the second order, the expression of the classical quasistatic effective stiffness tensor is recovered by $\boldsymbol{Z}^{[2]}$. Generally speaking, for $R \geq 1, \boldsymbol{Z}^{(2 R)}$ and $\boldsymbol{Z}^{[2 R]}$ agree over all terms of order lower 
than $R-1$ (these are the coefficients of all derivatives in the approximate effective motion equation up to order $R+1$ ).

Fourth, in order to define the effective $4^{\text {th }}$ order medium, the momentum balancebased approach uses more information since it requires to solve for $\tilde{\boldsymbol{u}}^{r}$ up to $r=3$ whereas to define $\boldsymbol{Z}^{[2]}$ only the solutions of order $r=0$ and $r=1$ are needed. It is indeed surprising that a $4^{\text {th }}$ order behavior can be defined with only a first order approximation of the microscopic solution. Smyshlyaev and Cherednichenko (2000) assessed however the quality of the approximation in a variational sense. What should be stressed is that said variational sense is different from the usual asymptotic one. We refer to the cited paper for details.

Finally, there are other approaches in the literature seeking to define a straingradient behavior from microstructures and crystal structures in particular. We mention the works of DiVincenzo (1986) and Maranganti and Sharma (2007). They identified the coefficients of an enriched Lagrangian thanks to computing the dispersion curve. Their method has however one important shortcoming since it is completely based on free waves propagation. The implications are that the effective mass density is postulated to be the mass per unit volume and that micro-inertia terms can never appear. In fact, their approach is fundamentally different and cannot be obtained through the present formalism since they have not clearly defined what the effective displacement field must be.

\section{Dispersion relation}

In this section, some general aspects of free wave propagation and dispersion curves for both Willis and LW-LF effective behaviors are studied. In particular, we explain why Willis theory yields exactly the whole microscopic dispersion curve while LW-LF asymptotics are restricted to acoustic branches. A theorem, in 1D setting, will be proven, showing that all optical branches are systematically lost when using LW-LF Taylor asymptotics. Optical branches are particularly interesting while studying metamaterials since they are responsible ${ }^{4}$ for desired non-standard effects such as "damping" (Liu et al., 2000). LW-LF Taylor asymptotics are therefore not rich enough to model these branches. Finally, we make a short detour by high-contrast and LW-FF asymptotics which allow to capture some optical modes.

\footnotetext{
${ }^{4}$ it is the band gap between acoustic and optical branches more precisely.
} 


\subsection{Exact considerations}

We will call a microscopic eigenmode, a mode $(\boldsymbol{k}, \omega)$ which allows for a free wave or a non-null solution, under $\boldsymbol{f}=\mathbf{0}$, to the microscopic motion equation (2.4). Effective eigenmodes are similarly defined with respect to the effective motion equation (2.6). Willis theory transforms a solution $\tilde{\boldsymbol{u}}_{\boldsymbol{k}, \omega}$ of (2.4) into a solution $\tilde{\boldsymbol{U}}_{\boldsymbol{k}, \omega}$ of (2.6) under the same loading. In particular, free waves are transformed into free waves. Therefore, microscopic and effective eigenmodes are the same except for particular modes when $\tilde{\boldsymbol{U}}_{\boldsymbol{k}, \omega}$ happens to be null in which case a microscopic freewave vanishes on the effective scale. To sum up, if the microscopic dispersion curve, i.e., the set of all microscopic eigenmodes, is symbolized by $\mathscr{C}$, and the effective one is denoted by $\mathscr{C}^{e}$ then we have

$$
\mathscr{C}^{e}=\mathscr{C}-\mathscr{C}^{\mathrm{za}}
$$

with

$$
\begin{aligned}
\mathscr{C}^{e} & =\left\{(\boldsymbol{k}, \omega) \in \mathscr{E}^{*} \times \mathbb{R} \text { such that } \operatorname{det} \boldsymbol{Z}_{\boldsymbol{k}, \omega}=0\right\}, \\
\mathscr{C}^{\mathrm{za}} & =\left\{(\boldsymbol{k}, \omega) \in \mathscr{C} \text { such that all solutions of }(2.4) \text { satisfy }\left\langle\tilde{\boldsymbol{u}}_{\boldsymbol{k}, \omega}\right\rangle=\mathbf{0}\right\},
\end{aligned}
$$

where "za" stands for "zero average". The elements of $\mathscr{C}^{\text {za }}$ correspond to the microscopic free waves disappearing in the effective description. Note that a continuous extrapolation of $\mathscr{C}^{e}$ over gaps caused by $\mathscr{C}^{\text {za }}$, if any, allows to recover $\mathscr{C}$ entirely, if said gaps were of empty interior. In this sense, microscopic dispersion curve $\mathscr{C}$ is characterized by the effective behavior.

Curve $\mathscr{C}$ has generally (for a periodically inhomogeneous continuum) an infinite number of branches $\omega=\omega_{n}(\boldsymbol{k})$ indexed with $n \in \mathbb{N}$. Branches which go through $(\boldsymbol{k}=\mathbf{0}, \omega=0)$ are called acoustic branches whereas all others are called optical branches. As noted earlier, it is these optical branches that produce many of the peculiar characteristics of metamaterials.

\section{2. $L W-L F$ approximations}

The approximate dispersion relation is naturally defined through

$$
\left\{(\boldsymbol{k}, \omega) \in \mathscr{E}^{*} \times \mathbb{R}^{+}, \quad \operatorname{det} \boldsymbol{Z}_{\boldsymbol{k}, \omega}^{(R)}=0\right\} .
$$

The lowest order approximation of the dispersion relation is based on $\boldsymbol{Z}^{0}$ from (4.4) and given by

$$
\operatorname{det}\left(-\omega^{2}\langle\rho\rangle \boldsymbol{I}+\boldsymbol{k} \cdot \boldsymbol{C}_{\mathbf{0}, 0}^{e} \cdot \boldsymbol{k}\right)=0 .
$$

Such curve has three branches $\omega=\omega_{n}^{(0)}(\boldsymbol{k})$ with $n \in\{0,1,2\}$, and all are acoustic. We underline the fact that the $\omega_{n}^{(0)}$ are positively homogeneous functions of degree 1: $\omega_{n}^{(0)}(a \boldsymbol{k})=|a| \omega_{n}^{(0)}(\boldsymbol{k})$. This implies that all waves, of sheet $n$, which propagate 
in direction $\boldsymbol{k} /\|\boldsymbol{k}\|$ have the same speed regardless of their wavelengths. In other words, the approximate effective behavior described by $\boldsymbol{Z}^{0}$ is non-dispersive, as is well known.

The classical homogenization limit has therefore two limitations: no dispersive effects and no optical branches can be captured. Dispersive effects can be however modeled by taking into account higher order approximations: $\boldsymbol{Z}^{(R)}, R>0$, which yield non-homogeneous functions $\omega=\omega_{n}^{(R)}(\boldsymbol{k}), n \in\{0,1,2\}$, and, correspondingly, a weakly non-local behavior. LW-LF asymptotics of order $R$ are still unable to approximate the optical branches whatever $R$ is. This intrinsic limitation of LW-LF asymptotics to acoustic branches is proved by the following theorem.

\subsection{Convergence domain}

Generally speaking, if the assumptions made for defining a scaling (see table 1) are not strictly satisfied, the approximate effective behavior will not have the expected accuracy. To improve accuracy, we can increase the truncation order $R$ or make better suited assumptions (change the scaling $p=p^{\epsilon}$ altogether). Increasing the order $R$ augments the accuracy of the approximate effective behavior over some neighborhood, or convergence domain, about $p=p_{i}(\epsilon=0)$. However, increasing $R$ does not change this domain. In order to change it, we have to improve the scaling or the path $p=p^{\epsilon}$.

Here, we prove a theorem, for arbitrary 1D media, stating that LF and FF Taylor expansions convergence domains are limited to a neighborhood of one branch, at most. Further, we demonstrate that LW-LF asymptotics systematically miss all optical branches. The keystone is Cauchy's theorem on the convergence domain of Taylor expansions of holomorphic functions. We start with general $d$ dimensional considerations and subsequently restrict our results to $d=1$. In particular, a general series representation of the Green operator of the effective medium will be useful and is derived first of all.

Let $\boldsymbol{k}$ be a given wavenumber. It is known (Bensoussan et al., 1978) that the eigenvalue problem:

$$
(\boldsymbol{\nabla}+i \boldsymbol{k}) \cdot\left\{\boldsymbol{C}(\boldsymbol{x}):\left[(\boldsymbol{\nabla}+i \boldsymbol{k}) \otimes{ }^{s} \boldsymbol{\psi}(\boldsymbol{x})\right]\right\}=-\omega^{2} \rho(\boldsymbol{x}) \boldsymbol{\psi}(\boldsymbol{x}),
$$

under periodic boundary conditions, admits a series of solutions $\omega=\omega_{n} \geq 0$, with $n \in \mathbb{N}$, which can be listed in an increasing order. To each eigenvalue, there corresponds an eigenvector $\boldsymbol{\psi}_{n}$. Given the solution $\tilde{\boldsymbol{u}}_{\boldsymbol{k}, \omega}$ to equation (2.4), we can, in view of the virtual work theorem, write

$$
\left\langle\boldsymbol{\psi}_{n}^{*} \cdot \tilde{\boldsymbol{f}}\right\rangle=\left(\omega_{n}^{2}-\omega^{2}\right)\left\langle\boldsymbol{\psi}_{n}^{*} \cdot \rho \tilde{\boldsymbol{u}}_{\boldsymbol{k}, \omega}\right\rangle .
$$


In particular, for $\tilde{\boldsymbol{f}}=\mathbf{0}, \omega=\omega_{m}$ and $\tilde{\boldsymbol{u}}_{\boldsymbol{k}, \omega}=\boldsymbol{\psi}_{m}$, we have the orthogonality property:

$$
\left(\omega_{n}^{2}-\omega_{m}^{2}\right)\left\langle\boldsymbol{\psi}_{n}^{*} \cdot \rho \boldsymbol{\psi}_{m}\right\rangle=0,
$$

which means that, with respect to the scalar product weighted with $\rho$, the family $\left\{\boldsymbol{\psi}_{n}\right\}_{n \in \mathbb{N}}$ can be assumed orthogonal. When normalized, said family becomes orthonormal which we assume hereafter. In fact, the family $\left\{\boldsymbol{\psi}_{n}\right\}_{n \in \mathbb{N}}$ forms an orthonormal basis for the space of solutions $\tilde{\boldsymbol{u}}_{\boldsymbol{k}, \omega}$. Accordingly, we have the following series representation of $\tilde{\boldsymbol{u}}_{\boldsymbol{k}, \omega}$ :

$$
\tilde{\boldsymbol{u}}_{k, \omega}=\sum_{n \in \mathbb{N}}\left\langle\boldsymbol{\psi}_{n}^{*} \cdot \rho \tilde{\boldsymbol{u}}_{\boldsymbol{k}, \omega}\right\rangle \boldsymbol{\psi}_{n},
$$

which, in view of (5.4), can be recast as

$$
\tilde{\boldsymbol{u}}_{\boldsymbol{k}, \omega}=\sum_{n \in \mathbb{N}} \frac{\left\langle\boldsymbol{\psi}_{n}^{*} \cdot \tilde{\boldsymbol{f}}\right\rangle}{\omega_{n}^{2}-\omega^{2}} \boldsymbol{\psi}_{n}=\sum_{n \in \mathbb{N}} \frac{\boldsymbol{\psi}_{n} \otimes\left\langle\boldsymbol{\psi}_{n}^{*}\right\rangle}{\omega_{n}^{2}-\omega^{2}} \cdot \tilde{\boldsymbol{f}}
$$

since $\tilde{\boldsymbol{f}}$ is a constant vector. Upon averaging, we get an expression for the effective displacement field:

$$
\tilde{\boldsymbol{U}}_{k, \omega}=\sum_{n \in \mathbb{N}} \frac{\left\langle\boldsymbol{\psi}_{n}\right\rangle \otimes\left\langle\boldsymbol{\psi}_{n}^{*}\right\rangle}{\omega_{n}^{2}-\omega^{2}} \cdot \tilde{\boldsymbol{f}},
$$

and, most importantly, an expression for the inverse of the effective impedance $\boldsymbol{G}_{\boldsymbol{k}, \boldsymbol{\omega}}$ :

$$
\boldsymbol{G}_{\boldsymbol{k}, \omega} \equiv \boldsymbol{Z}_{\boldsymbol{k}, \omega}^{-1}=\sum_{n \in \mathbb{N}} \frac{\left\langle\boldsymbol{\psi}_{n}\right\rangle \otimes\left\langle\boldsymbol{\psi}_{n}^{*}\right\rangle}{\omega_{n}^{2}-\omega^{2}} .
$$

Note that all $\boldsymbol{\psi}_{n}$ and $\omega_{n}$ are $\boldsymbol{k}$-dependent and $\omega$-independent. The second order tensor $\boldsymbol{G}_{\boldsymbol{k}, \omega}$ is the Green operator, in Fourier domain, of the effective medium. A corresponding expression for $\boldsymbol{Z}_{k, \omega}$ can be derived using Cramer's rule for instance. This is not done here since we shall consider $d=1$ next.

For 1D media, the effective impedance and Green tensors become scalars. In particular, we have

$$
G_{k, \omega}=\sum_{n \in \mathbb{N}} \frac{\left|\left\langle\psi_{n}\right\rangle\right|^{2}}{\omega_{n}^{2}-\omega^{2}} .
$$

We now assume that the eigenvalues $\omega_{n}$ are simple, for clarity purposes. Consider the limits of $G_{k, \omega}$ as $\omega \rightarrow \omega_{n}^{+}$and $\omega \rightarrow \omega_{n+1}^{-}$for two consecutive eigen frequencies $\omega_{n}$ and $\omega_{n+1}$ with $\omega_{n}<\omega_{n+1}$. It can be seen that:

$$
G_{k, \omega_{n}^{+}}=-\infty \quad \text { and } \quad G_{k, \omega_{n+1}^{-}}=+\infty
$$


given $\left|\left\langle\psi_{n}\right\rangle\right|^{2} \neq 0$ and $\left|\left\langle\psi_{n+1}\right\rangle\right|^{2} \neq 0$.

Now let $\epsilon \mapsto p^{\epsilon}=\left(k^{\epsilon}, \omega^{\epsilon}\right)$ be a path connecting one point from the $n^{\text {th }}$ branch, denoted by $\left(k^{\epsilon_{n}}, \omega_{n}\left(k^{\epsilon_{n}}\right)\right)$ and attained for $\epsilon=\epsilon_{n}>0$, to another from branch $n+1$, called $\left(k^{\epsilon_{n+1}}, \omega_{n+1}\left(k^{\epsilon_{n+1}}\right)\right)$ and reached for $\epsilon=\epsilon_{n+1}>\epsilon_{n}$. Without loss of generality, we assume that $p^{\epsilon}$ remains between the two branches $n$ and $n+1$. Recalling that $Z_{k, \omega}=1 / G_{k, \omega}$, the question is whether a Taylor expansion of $Z^{\epsilon}=Z_{k^{\epsilon}, \omega^{\epsilon}}$ can converge at, and recover, both eigenmodes $\left(k^{\epsilon_{n}}, \omega_{n}\left(k^{\epsilon_{n}}\right)\right)$ and $\left(k^{\epsilon_{n+1}}, \omega_{n+1}\left(k^{\epsilon_{n+1}}\right)\right)$.

First of all, if either $\left|\left\langle\psi_{n}\left(k=k^{\epsilon_{n}}\right)\right\rangle\right|^{2}$ or $\left|\left\langle\psi_{n+1}\left(k=k^{\epsilon_{n+1}}\right)\right\rangle\right|^{2}$ is null then the answer is no since $Z_{k, \omega}$ is, already itself, unable to recover the corresponding eigenmode according to (5.1). We assume then that both $\left|\left\langle\psi_{n}\left(k=k^{\epsilon_{n}}\right)\right\rangle\right|^{2}$ and $\left|\left\langle\psi_{n+1}\left(k=k^{\epsilon_{n+1}}\right)\right\rangle\right|^{2}$ are non-null. Then, thanks to (5.7), $G^{\epsilon}=G_{k^{\epsilon}, \omega^{\epsilon}}$ changes sign when $\epsilon$ passes from $\epsilon_{n}$ to $\epsilon_{n+1}$ and attains, by continuity, zero for some value of $\epsilon$ in $] \epsilon_{n}, \epsilon_{n+1}[$. The existence of a zero value for $G^{\epsilon}$ implies that $Z^{\epsilon}$ explodes at some point on the path $p^{\epsilon}$ between $\epsilon_{n}$ and $\epsilon_{n+1}$. This singularity of $Z^{\epsilon}$, by Cauchy's theorem, forbids convergence of Taylor expansion of $Z^{\epsilon}$ about $\epsilon=0 \leq \epsilon_{n}$ at $\epsilon=\epsilon_{n+1}$.

In particular, LW-LF Taylor asymptotics can converge only near the acoustic branch. Optical branches are systematically lost. LW-FF Taylor asymptotics converge near one optical branch at one time. For higher dimensions, singularities seem also to limit the convergence domain although not in the nice one-branch-at-a-time manner (a weaker version of the above theorem holds but is not presented here).

In order to have a Taylor expansion valid for multiple branches, one needs a path $p=p^{\epsilon}$ which avoids the singularities of $Z$. Needless to say that such a path will have to get out of the $(k, \omega)$-plane and surf other dimensions. High-contrast scalings provide, sometimes, depending on the underlying microstructure, such paths. A LW-LF-high-contrast scaling can hence extend the convergence domain to englobing multiple acoustic and optical branches. We refer to papers by Auriault and Bonnet (1985) and by Auriault and Boutin (2012) for examples of such scalings.

Here, it is the definition of the approximate effective impedance in the momentum balance-based approach that is adopted: $\boldsymbol{Z}^{(R)}$. Similar results for $\boldsymbol{Z}^{[R]}$ are thought to hold.

\subsection{Remark: on tuning}

The loss of optical branches means that there could exist solutions to the approximate dispersion equation with complex $\omega$. Such solutions are unstable and non-physical. A "tuning" technique, due to Pichugin et al. (2008), introduces artificial optical branches so that the approximate motion equation becomes stable. However, the optical modes thus introduced still have no physical meaning. In addition, note that a tuned motion equation is an implicit motion equation in the sense that deriva- 
tives of $\boldsymbol{f}$ appear when writing the tuned motion equation in forced regime. This explains how tuned approximate motion equations are not unique while the partial sums $\boldsymbol{Z}^{(R)}$, and accordingly equation (4.5), are unique by the uniqueness of Taylor expansions.

\subsection{On high-contrast and $L W-F F$ asymptotic approximations}

As observed earlier, in order to approximate any optical branch of the dispersion curve, the LW-LF scaling must be abandoned in favor of other scalings such as the high-contrast or the LW-FF ones first investigated by Auriault and Bonnet (1985) and by Daya et al. (2002), respectively. In common with these two approaches is the fact that, to the lowest order, the displacement field can be written as a large scale modulation $D$ of an $\mathscr{R}$-periodic shape or weighting function $\phi$ :

$$
\boldsymbol{u}\left(\boldsymbol{x}, \boldsymbol{x}^{\prime}\right)=\phi\left(\boldsymbol{x}^{\prime}\right) D(\boldsymbol{x}) .
$$

Both $\phi$ and $D$ can be either scalars, vectors or tensors but their product, in the corresponding sense, must yield a vector. For a highly contrasted hard matrix-soft inclusion composite, $\phi$ turns out to be the indicator function of the matrix phase whereas for the LW-FF scaling, $\phi$ is an $\mathscr{R}$-periodic free oscillation mode of the microstructure. In both cases, $D$ appears as the natural macroscopic displacement field as it depends only on the macroscopic variable $\boldsymbol{x}$. In contrast, Willis theory as presented above defines the macroscopic displacement as

$$
\boldsymbol{U}(\boldsymbol{x})=\langle\phi\rangle D(\boldsymbol{x}) .
$$

Unfortunately, in some cases ${ }^{5},\langle\phi\rangle$ can induce a non-negligible error making $\boldsymbol{U}$ an inappropriate approximation of $\boldsymbol{u}$.

Independently, a modified Willis theory which defines the macroscopic displacement field as the weighted average

$$
\boldsymbol{U}^{w}(\boldsymbol{x})=\left\langle w\left(\boldsymbol{x}^{\prime}\right) \boldsymbol{u}\left(\boldsymbol{x}, \boldsymbol{x}^{\prime}\right)\right\rangle_{\boldsymbol{x}^{\prime}}
$$

for a given weight function $w$ was suggested by Milton and Willis (2007). It is then remarkable that by appropriately choosing $w$ so that the averaged product $\langle w \phi\rangle$ becomes equal to the identity, we can recover $\boldsymbol{U}^{w}=D$. Note that introducing the weight $w$ does not essentially modify any of the formal aspects of Willis theory (Willis, 2011). Therefore, the frameworks of high-contrast and LW-FF asymptotics remain consistent with Willis theory and can benefit from the same connections established in detail in the previous sections for LW-LF asymptotics.

\footnotetext{
${ }^{5}$ The worst scenario occurs for a thin walled matrix when $\phi$ is the matrix indicator function and for a homogeneous mass density $\rho$ when $\phi$ is an $\mathscr{R}$-periodic optical mode.
} 


\section{Example: a 1D string}

In this section, the exact effective impedance $Z$ of a two-phase $1 \mathrm{D}$ string is calculated, scaled and expanded in order to exemplify what has been presented in the previous sections.

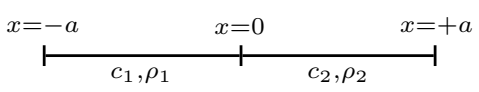

Figure 1: A unit cell of a 1D 2-phase string.

\subsection{Setup and exact effective behavior}

The periodic 1D string to be studied has two phases of stiffness $c_{j}$ and mass density $\rho_{j}$ with $j \in\{1,2\}$. A unit cell of length $2 a$ is illustrated by figure 1 . The waves propagating along the string are either transverse or longitudinal waves (but not both since this is a $1 \mathrm{D}$ example).

The motion equation (2.3) reduces, over each phase, to

$$
c_{j} u^{\prime \prime}+\tilde{f} e^{i k x}=-\omega^{2} \rho_{j} u,
$$

where the superscript $\left({ }^{\prime}\right)$ stands for $\mathrm{d} / \mathrm{d} x$ and $\tilde{f}$ is the amplitude of body forces and is constant. The displacement $u$ and traction $C u^{\prime}$ are required to be continuous across phases and to satisfy the Bloch condition: $u(x) e^{-i k x}=\tilde{u}(x)$ and

$$
\left[C u^{\prime}\right](x) e^{-i k x}=C(x)\left(\tilde{u}^{\prime}(x)+i k \tilde{u}(x)\right)
$$

are periodic functions of period $2 a$.

Once $u$ is calculated, the effective impedance $Z$ can be defined as $Z=\tilde{f} /\langle\tilde{u}\rangle$ and is a function, exclusively, of the period half-length $a$, stiffnesses $c_{1}$ and $c_{2}$, mass densities $\rho_{1}$ and $\rho_{2}$, frequency $\omega$ and wavenumber $k$. The general form of the solution $u$ for phase $j$ is known to be

$$
u(x)=B_{j}^{+} \exp \left(i \omega \sqrt{\rho_{j} / c_{j}} x\right)+B_{j}^{-} \exp \left(-i \omega \sqrt{\rho_{j} / c_{j}} x\right)+\frac{\tilde{f}}{c_{j} k^{2}-\rho_{j} \omega^{2}} \exp (i k x),
$$

where the $B_{j}^{+,-}$are constants to be identified by the continuity and Bloch conditions. Note that the above equation is valid as long as pairs $(k, \omega)$ satisfying $c_{j} k^{2}-\rho_{j} \omega^{2} \neq 0$ are excluded. 
The identification of the constants presents no particular interest for our purpose and is skipped here. We directly give a closed form analytical expression for $Z$ as

$$
Z=P / Q
$$

where

$$
\begin{aligned}
& P=a\left(c_{1} k^{2}-\rho_{1} \omega^{2}\right)^{2}\left(c_{2} k^{2}-\rho_{2} \omega^{2}\right)^{2}\left\{4 \sqrt{c_{1} \rho_{1} c_{2} \rho_{2}} \cos (2 k a)\right. \\
&+\left(\sqrt{c_{1} \rho_{1}}-\sqrt{c_{2} \rho_{2}}\right)^{2} \cos \left[\omega\left(\sqrt{\rho_{1} / c_{1}}-\sqrt{\rho_{2} / c_{2}}\right) a\right] \\
&\left.\quad-\left(\sqrt{c_{1} \rho_{1}}+\sqrt{c_{2} \rho_{2}}\right)^{2} \cos \left[\omega\left(\sqrt{\rho_{1} / c_{1}}+\sqrt{\rho_{2} / c_{2}}\right) a\right]\right\},
\end{aligned}
$$

and where $Q$ is specified in AppendixB. Note that $Z$ can be continuously extrapolated over the previously excluded values of $k$ and $\omega$. The dependence of $Z$ on $k$ and $\omega$ is complicated and cannot be easily interpreted. What is certain is that $Z$ describes a non-local effective behavior in both space and time since it is neither a polynomial in $k$ nor in $\omega$.

The dispersion relation, as can be found in Andrianov et al. (2008) and references therein, is given by

$$
\begin{aligned}
\cos (2 k a)= & \frac{\left(\sqrt{c_{1} \rho_{1}}+\sqrt{c_{2} \rho_{2}}\right)^{2}}{4 \sqrt{c_{1} \rho_{1} c_{2} \rho_{2}}} \cos \left[\omega\left(\sqrt{\rho_{1} / c_{1}}+\sqrt{\rho_{2} / c_{2}}\right) a\right] \\
& -\frac{\left(\sqrt{c_{1} \rho_{1}}-\sqrt{c_{2} \rho_{2}}\right)^{2}}{4 \sqrt{c_{1} \rho_{1} c_{2} \rho_{2}}} \cos \left[\omega\left(\sqrt{\rho_{1} / c_{1}}-\sqrt{\rho_{2} / c_{2}}\right) a\right] .
\end{aligned}
$$

This is the same as $P=0$ up to the $\left(c_{j} k^{2}-\rho_{j} \omega^{2}\right)^{2}$ factors. In fact, the dispersion relation can be obtained as argued before from $Z=\operatorname{det}(Z)=0$ and not $P=0$ since zeros of $P$ can be "canceled" by zeros of $Q$. In particular, whenever $\left(c_{j} k^{2}-\rho_{j} \omega^{2}\right)^{2}$ is null, $Q$ is null. This is not obvious and can be checked through calculations. Note that the dispersion curve is even, periodic ${ }^{6}$ in $k$ and of period $\pi / a$.

A typical dispersion curve is plotted in figure 2. It shows the first three branches of the dispersion curve $Z=0: \omega_{n}(k)$ with $n \in\{1,2,3\}$; the first two branches of the curve $1 / Z=0$ are also displayed: $\varpi_{n}(k)$ with $n \in\{1,2\}$. We can see, as implied by our theorem, that $\omega_{1} \leq \varpi_{1} \leq \omega_{2} \leq \varpi_{2} \leq \omega_{3} \ldots$ In the plot, wavenumbers have been normalized by a factor of $1 / a$ and frequencies $\omega$ by a factor $\omega_{o}=\frac{\pi}{2 a \sqrt{\langle 1 / C\rangle\langle\rho\rangle}}$. The

\footnotetext{
${ }^{6}$ Incidentally, the dispersion curve is also periodic with the frequency $\omega$ if and only if the expression $\frac{\sqrt{\rho_{1} / c_{1}}-\sqrt{\rho_{2} / c_{2}}}{\sqrt{\rho_{1} / c_{1}}+\sqrt{\rho_{2} / c_{2}}}$ is a rational number.
} 


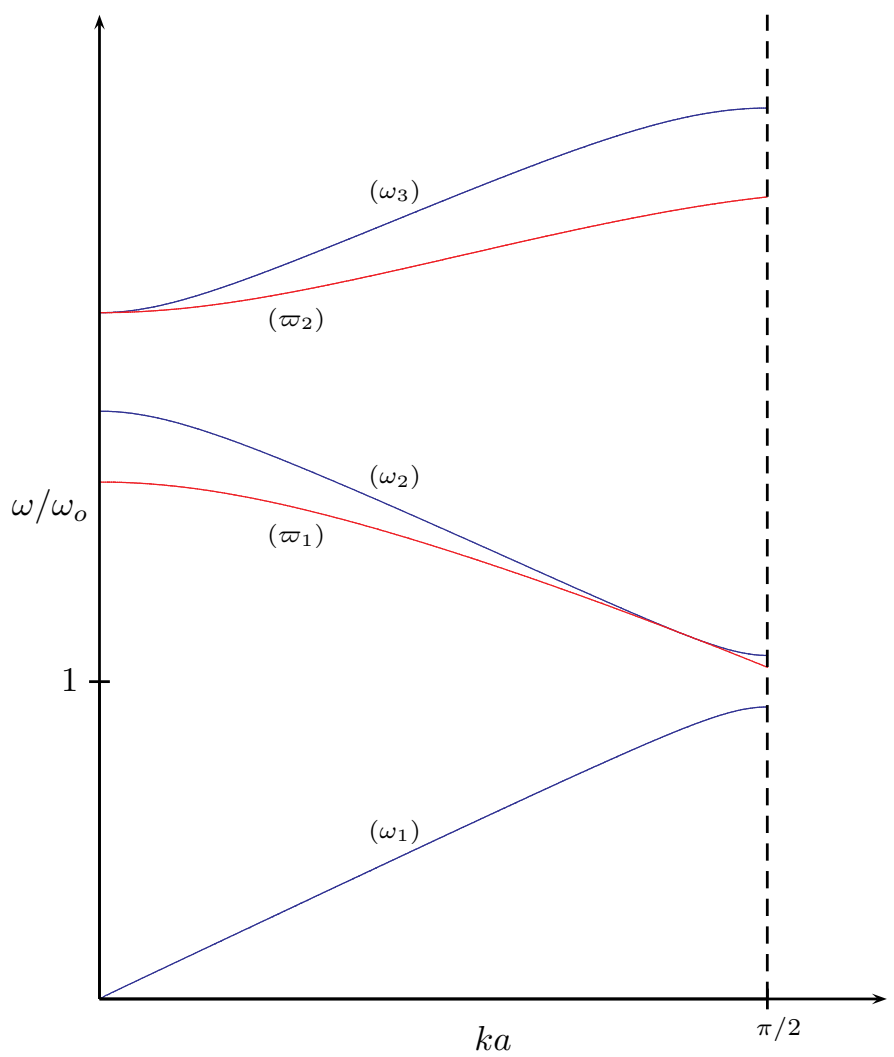

Figure 2: A typical dispersion curve. The first three branches, $\left(\omega_{1}\right),\left(\omega_{2}\right)$ and $\left(\omega_{3}\right)$, are plotted against the first two branches of singularities of the effective impedance $Z, \varpi_{1}$ and $\varpi_{2}$. The dashed line marks off the first Brillouin zone boundary.

numerical values used in the plots are:

$$
c_{1}=10^{10} \mathrm{~N}, c_{2}=4.10^{12} \mathrm{~N}, \rho_{1}=4900 \mathrm{~kg} / \mathrm{m}, \rho_{2}=900 \mathrm{~kg} / \mathrm{m}, a=5.10^{-3} \mathrm{~m} \text {. }
$$

\section{2. $L W$-LF asymptotics}

We next explore second order expansions of the Willis impedance $Z$ according to the two approaches discussed earlier and make a brief comparison with Mindlin's 1D second-order strain-gradient medium. 


\subsubsection{Momentum balance-based approach}

Define

$$
Z^{\epsilon}=\frac{1}{\epsilon^{2}} Z_{\epsilon k, \epsilon \omega}
$$

as in (4.3). The lowest order approximation to $Z$ is therefore

$$
Z^{(0)}=Z^{0}=\lim _{\epsilon \rightarrow 0} Z^{\epsilon}=2 \frac{c_{1} c_{2}}{c_{1}+c_{2}} k^{2}-\frac{\rho_{1}+\rho_{2}}{2} \omega^{2},
$$

as expected. An approximation of order 2 has the form

$$
\begin{aligned}
& Z^{(2)}=2 \frac{c_{1} c_{2}}{c_{1}+c_{2}} k^{2}-\frac{\rho_{1}+\rho_{2}}{2} \omega^{2} \\
& -\frac{a^{2}}{24} \frac{\left(c_{1}-c_{2}\right)\left[2\left(\rho_{1} c_{1}-\rho_{2} c_{2}\right)+\left(c_{1}+c_{2}\right)\left(\rho_{1}-\rho_{2}\right)\right]}{\left(c_{1}+c_{2}\right)^{2}} \omega^{2} k^{2} \\
& -\frac{a^{2}}{96}\left(\rho_{1}-\rho_{2}\right)^{2} \frac{\left(c_{1}+c_{2}\right)}{c_{1} c_{2}} \omega^{4} .
\end{aligned}
$$

The corresponding approximate effective motion equation, in the real space-time domain, has the expression

$$
\begin{aligned}
&-2 \frac{c_{1} c_{2}}{c_{1}+c_{2}} u^{\prime \prime}(x, t)+\frac{\rho_{1}+\rho_{2}}{2} \ddot{u}(x, t) \\
&-\frac{a^{2}}{24} \frac{\left(c_{1}-c_{2}\right)\left[2\left(\rho_{1} c_{1}-\rho_{2} c_{2}\right)+\left(c_{1}+c_{2}\right)\left(\rho_{1}-\rho_{2}\right)\right]}{\left(c_{1}+c_{2}\right)^{2}} \ddot{u}^{\prime \prime}(x, t) \\
& \quad-\frac{a^{2}}{96}\left(\rho_{1}-\rho_{2}\right)^{2} \frac{\left(c_{1}+c_{2}\right)}{c_{1} c_{2}} \ddot{u}(x, t)=f(x, t),
\end{aligned}
$$

where the superscripted dot stands for $\mathrm{d} / \mathrm{d} t$. Note that there is no term in $u^{\prime \prime \prime \prime}$. This means that there are no purely spatial higher order strain-gradient effects. In 1D, this is not a particular feature of the approximation order. In fact, for all similar 1D systems, we have in statics:

$$
Z_{\omega=0}=2 \frac{c_{1} c_{2}}{c_{1}+c_{2}} k^{2}
$$

Higher order strain gradient effects, and general strong non-local effects, caused by heterogeneities appear only under dynamic loadings or when homogenizing in higher dimensions (2D and 3D layered composites for instance), at least according to Willis and equivalent theories. Strain gradient effects do appear in LW asymptotics when continualizing discrete 1D lattices (see for example Pichugin et al. 2008). At this stage, continualization of discrete lattices and homogenization of already continuous media need to be clearly distinguished. 


\subsubsection{Energy-based approach}

It is easy to see that the first order localization tensor is given by

$$
A(y)=\left(\frac{1}{C(y)}-\left\langle\frac{1}{C}\right\rangle\right) \frac{y}{\langle 1 / C\rangle}-\frac{a}{2} \frac{c_{1}-c_{2}}{c_{1}+c_{2}} .
$$

When this expression is injected into (4.12), we get

$$
Z^{[2]}=2 \frac{c_{1} c_{2}}{c_{1}+c_{2}} k^{2}-\frac{\rho_{1}+\rho_{2}}{2} \omega^{2}-\frac{a^{2}}{24} \frac{\left(c_{1}-c_{2}\right)^{2}\left(\rho_{1}+\rho_{2}\right)}{\left(c_{1}+c_{2}\right)^{2}} \omega^{2} k^{2}+\frac{a^{2}}{24} \frac{\left(c_{1}-c_{2}\right)^{2}}{c_{1}+c_{2}} k^{4} .
$$

As expected, no $\omega^{4}$ terms appear. However, the $k^{4}$ coefficient is non-null contrarily to what happens in the momentum balance-based approach. Therefore, for higher order approximations, we expect the coefficient of $k^{4}$ to be corrected and eventually to be set to zero (the same goes for all $k^{n}$ with $n>2$, according to (6.3)).

\subsubsection{Signature}

In order to compare both effective models with Mindlin's theory, it is convenient to define a signature which is the series of signs of the coefficients appearing in the expression of the effective impedance. The results are as follows:

$$
\begin{aligned}
& \begin{array}{lllll}
k^{2} & \omega^{2} & k^{2} \omega^{2} & k^{4} & \omega^{4}
\end{array} \\
& \text { Mindlin (1964) }+\quad-\quad-\quad+0 \\
& \text { momentum balance-based approach }+-+ \text { or }-0 \text { - } \\
& \text { energy-based approach }+-{ }_{-}+0
\end{aligned}
$$

It is seen that the energy-based approach yields the same signature as in Mindlin's theory (for 1D media) whereas the momentum balance-based approach has generally a different signature.

\subsubsection{Approximate dispersion curve}

The approximate dispersion curves, $\omega_{1}^{(0)}(k)$ and $\omega_{1}^{(2)}(k)$, are calculated, according to (5.2) and have the equations:

$$
Z^{(0)}=0 \quad \text { and } \quad Z^{(2)}=0 .
$$

They are plotted in figure 3 and are almost indistinguishable. Note that these approximations have only acoustic branches as expected. As can be seen, they yield a good approximation for low frequencies (near the acoustic branch) and for almost all low wavelengths up to, and except near, the frontier of the first Brillouin zone. 
The same goes for the energy-based approach which defines an approximation to the dispersion curve, $\omega^{[2]}(k)$, of equation:

$$
Z^{[2]}=0
$$

Curve $\omega^{[2]}(k)$ is a poor approximation except in the quasistatic range near $\omega=0, k=0$ (see figure 3). As said earlier, the energy-based approach defines an approximate effective impedance in a variational sense and is based on the asymptotics of the localization operator. The momentum balance-based approach on the other hand relies on the Taylor series of $Z$ directly. Having $Z=\operatorname{det}(Z)$ in $1 \mathrm{D}$, it is understandable that the momentum balance-based approach yields a better approximation of the dispersion curve. In higher dimensions, similar observations are expected to hold even though a truncated Taylor series of $\boldsymbol{Z}$ does not produce a truncated Taylor series of $\operatorname{det}(\boldsymbol{Z})$. This is because the error committed therein remains smaller than the one committed by approximating $\operatorname{det}(\boldsymbol{Z})$ based on a truncated series of the localization operator.

\section{Conclusion}

Willis elastodynamic homogenization theory is general and exact, giving rise to an effective constitutive behavior which is non-local in both space and time. In the present work dedicated to periodic media, we have suggested the fundamental idea of using Willis theory as the basis for deriving a large class of asymptotic elastodynamic homogenization approaches through making appropriate assumptions on loadings, microstructure and phase property contrast. Having realized this idea, the present work has clarified the relations between Willis theory and a good few asymptotic theories reported in the literature, which were proposed and have been up to now developed independently. We also believe that, in doing so, the present work has contributed to getting new insights into Willis theory and asymptotic approaches, and to their respective development.

Andrianov, I.V., Bolshakov, V.I., Danishevs'kyy, V.V., Weichert, D., 2008. Higher order asymptotic homogenization and wave propagation in periodic composite materials. Proceedings of the Royal Society A: Mathematical, Physical and Engineering Sciences 464, 1181-1201.

Antonakakis, T., Craster, R.V., Guenneau, S., 2014. Homogenisation for elastic photonic crystals and dynamic anisotropy. Journal of the Mechanics and Physics of Solids 71, 84-96. 


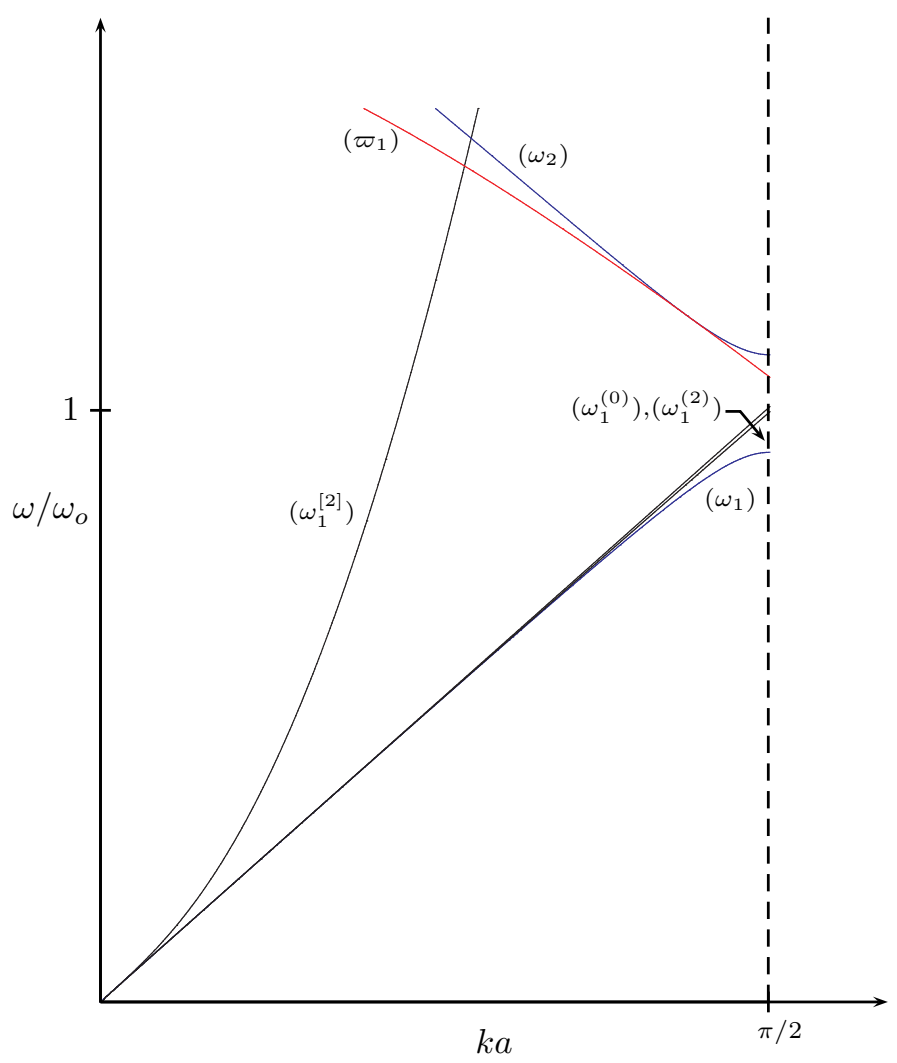

Figure 3: LW-LF approximations to the dispersion curve.

Auriault, J.L., Bonnet, G., 1985. Dynamics of periodic elastic composites (Dynamique des composites elastiques periodiques). Archiwum Mechaniki Stosowanej 37, 269284.

Auriault, J.L., Boutin, C., 2012. Long wavelength inner-resonance cut-off frequencies in elastic composite materials. International Journal of Solids and Structures 49, 3269-3281.

Bensoussan, A., Lions, J.L., Papanicolaou, G., 1978. Asymptotic analysis for periodic structures. North-Holland Publishing Company.

Boutin, C., 1996. Microstructural effects in elastic composites. International Journal of Solids and Structures 33, 1023-1051. 
Boutin, C., Auriault, J.L., 1993. Rayleigh scattering in elastic composite materials. International Journal of Engineering Science 31, 1669-1689.

Boutin, C., Rallu, A., Hans, S., 2014. Large scale modulation of high frequency waves in periodic elastic composites. Journal of the Mechanics and Physics of Solids 70, $362-381$.

Chen, H., Chan, C.T., 2010. Acoustic cloaking and transformation acoustics. Journal of Physics D: Applied Physics 43, 113001.

Colquitt, D.J., Craster, R.V., Antonakakis, T., Guenneau, S., 2014. Rayleigh-Bloch waves along elastic diffraction gratings. Proceedings of the Royal Society A: Mathematical, Physical and Engineering Sciences 471, 20140465.

Craster, R.V., Kaplunov, J., Pichugin, A.V., 2010. High-frequency homogenization for periodic media. Proceedings of the Royal Society A: Mathematical, Physical and Engineering Sciences 466, 2341-2362.

Daya, E., Braikat, B., Damil, N., Potier-Ferry, M., 2002. Continuum modeling for the modulated vibration modes of large repetitive structures. Comptes Rendus Mécanique 330, 333-338.

DiVincenzo, D.P., 1986. Dispersive corrections to continuum elastic theory in cubic crystals. Physical review B 34, 5450-5465.

Lee, J.H., Singer, J.P., Thomas, E.L., 2012. Micro-/nanostructured mechanical metamaterials. Advanced materials 24, 4782-4810.

Liu, Z., Zhang, X., Mao, Y., Zhu, Y.Y., Yang, Z., Chan, C.T., Sheng, P., 2000. Locally resonant sonic materials. Science (New York, N.Y.) 289, 1734-1736.

Maranganti, R., Sharma, P., 2007. A novel atomistic approach to determine straingradient elasticity constants: Tabulation and comparison for various metals, semiconductors, silica, polymers and the (Ir) relevance for nanotechnologies. Journal of the Mechanics and Physics of Solids 55, 1823-1852.

Milton, G.W., Briane, M., Willis, J.R., 2006. On cloaking for elasticity and physical equations with a transformation invariant form. New Journal of Physics 8, 248-267.

Milton, G.W., Willis, J.R., 2007. On modifications of Newton's second law and linear continuum elastodynamics. Proceedings of the Royal Society A: Mathematical, Physical and Engineering Sciences 463, 855-880. 
Milton, G.W., Willis, J.R., 2010. Minimum variational principles for time-harmonic waves in a dissipative medium and associated variational principles of HashinShtrikman type. Proceedings of the Royal Society A: Mathematical, Physical and Engineering Sciences 466, 3013-3032.

Mindlin, R.D., 1964. Micro-structure in linear elasticity. Archive for Rational Mechanics and Analysis 16, 51-78.

Nassar, H., He, Q.C., Auffray, N., 2015. Willis elastodynamic homogenization theory revisited for periodic media. Journal of the Mechanics and Physics of Solids 77, $158-178$.

Nemat-Nasser, S., Srivastava, A., 2011. Overall dynamic constitutive relations of layered elastic composites. Journal of the Mechanics and Physics of Solids 59, 1953-1965.

Nemat-Nasser, S., Srivastava, A., 2012. Homogenized dynamic constitutive relation for Bloch-wave propagation in periodic composites: structure and symmetries. Proceedings SPIE 8269, 82690Y-82690Y-9.

Nemat-Nasser, S., Srivastava, A., 2013. Bounds on effective dynamic properties of elastic composites. Journal of the Mechanics and Physics of Solids 61, 254-264.

Nemat-Nasser, S., Willis, J.R., Srivastava, A., Amirkhizi, A.V., 2011. Homogenization of periodic elastic composites and locally resonant sonic materials. Physical Review B 83, 104103.

Nolde, E., Craster, R.V., Kaplunov, J., 2011. High frequency homogenization for structural mechanics. Journal of the Mechanics and Physics of Solids 59, 651-671.

Norris, A.N., 2008. Acoustic cloaking theory. Proceedings of the Royal Society A: Mathematical, Physical and Engineering Sciences 464, 2411-2434.

Norris, A.N., Shuvalov, A.L., 2011. Elastic cloaking theory. Wave Motion 48, 525-538.

Norris, A.N., Shuvalov, A.L., Kutsenko, A.A., 2012. Analytical formulation of threedimensional dynamic homogenization for periodic elastic systems. Proceedings of the Royal Society A: Mathematical, Physical and Engineering Sciences 468, 1629-1651.

Pichugin, A.V., Askes, H., Tyas, A., 2008. Asymptotic equivalence of homogenisation procedures and fine-tuning of continuum theories. Journal of Sound and Vibration $313,858-874$. 
Sanchez-Palencia, E., 1980. Non-homogeneous media and vibration theory. SpringerVerlag.

Shuvalov, A.L., Kutsenko, A.A., Norris, A.N., Poncelet, O., 2011. Effective Willis constitutive equations for periodically stratified anisotropic elastic media. Proceedings of the Royal Society A: Mathematical, Physical and Engineering Sciences 467, 1749-1769.

Smyshlyaev, V.P., Cherednichenko, K.D., 2000. On rigorous derivation of strain gradient effects in the overall behaviour of periodic heterogeneous media. Journal of the Mechanics and Physics of Solids 48, 1325-1357.

Srivastava, A., Nemat-Nasser, S., 2011. Overall dynamic properties of threedimensional periodic elastic composites. Proceedings of the Royal Society A: Mathematical, Physical and Engineering Sciences 468, 269-287.

Turbé, N., 1982. Applications of Bloch expansion to periodic elastic and viscoelastic media. Mathematical Methods in the Applied Sciences 4, 433-449.

Turbé, N., 1989. Bloch expansion in generalized thermoelasticity. International Journal of Engineering Science 27, 55-62.

Willis, J.R., 1980a. A polarization approach to the scattering of elastic waves-I. Scattering by a single inclusion. Journal of the Mechanics and Physics of Solids 28, 287-305.

Willis, J.R., 1980b. A polarization approach to the scattering of elastic waves-II. Multiple scattering. Journal of the Mechanics and Physics of Solids 28, 307-327.

Willis, J.R., 1981. Variational principles for dynamic problems for inhomogeneous elastic media. Wave Motion 3, 1-11.

Willis, J.R., 1985. The nonlocal influence of density variations in a composite. International Journal of Solids and Structures 21, 805-817.

Willis, J.R., 1997. Dynamics of composites, in: Suquet, P. (Ed.), Continuum Micromechanics. Springer-Verlag New York, Inc., pp. 265-290.

Willis, J.R., 2009. Exact effective relations for dynamics of a laminated body. Mechanics of Materials 41, 385-393. 
Willis, J.R., 2011. Effective constitutive relations for waves in composites and metamaterials. Proceedings of the Royal Society A: Mathematical, Physical and Engineering Sciences 467, 1865-1879.

Willis, J.R., 2012. The construction of effective relations for waves in a composite. Comptes Rendus Mécanique 340, 181-192. 


\section{AppendixA. Willis effective constitutive law}

Under the loading of a (harmonic) plane wave body force $\boldsymbol{f}(\boldsymbol{x})=\tilde{\boldsymbol{f}} e^{i \boldsymbol{k} \cdot \boldsymbol{x}}$, all fields: displacement $\boldsymbol{u}$, velocity $\boldsymbol{v}$, strain $\boldsymbol{\varepsilon}$, momentum density $\boldsymbol{p}$, stress $\boldsymbol{\sigma}$, are Bloch waves of the form

$$
\boldsymbol{\phi}(\boldsymbol{x})=\tilde{\phi}(\boldsymbol{x}) e^{i \boldsymbol{k} \cdot \boldsymbol{x}}
$$

for $\boldsymbol{\phi} \in\{\boldsymbol{u}, \boldsymbol{v}, \boldsymbol{\varepsilon}, \boldsymbol{p}, \boldsymbol{\sigma}\}$. The corresponding effective fields $\boldsymbol{\Phi} \in\{\boldsymbol{U}, \boldsymbol{V}, \boldsymbol{E}, \boldsymbol{P}, \boldsymbol{\Sigma}\}$ are defined by

$$
\boldsymbol{\Phi}(\boldsymbol{x}) \equiv \tilde{\boldsymbol{\Phi}} e^{i \boldsymbol{k} \cdot \boldsymbol{x}} \equiv\langle\tilde{\boldsymbol{\phi}}\rangle e^{i \boldsymbol{k} \cdot \boldsymbol{x}} .
$$

Then, the effective fields are related to one another according to the following effective constitutive law (Willis, 2011)

$$
\left[\begin{array}{l}
\boldsymbol{\Sigma} \\
\boldsymbol{P}
\end{array}\right]=\left[\begin{array}{ll}
\boldsymbol{C}^{e} & \boldsymbol{S}^{1} \\
\boldsymbol{S}^{2} & \boldsymbol{\rho}^{e}
\end{array}\right]_{k, \omega}\left[\begin{array}{l}
\boldsymbol{E} \\
\boldsymbol{V}
\end{array}\right],
$$

where $\boldsymbol{S}^{1}$ is a stress-velocity coupling tensor of order 3 and $\boldsymbol{S}^{2}$ is a momentum density-strain coupling tensor of order 3 . The effective motion equation is

$$
i \boldsymbol{k} \cdot \boldsymbol{\Sigma}+\boldsymbol{f}=i \omega \boldsymbol{P}
$$

Combined with the effective constitutive law, the above equation leads to equation (2.7) where tensor $\boldsymbol{S}$ has been defined by

$$
\boldsymbol{S} \equiv \boldsymbol{S}^{2}-\boldsymbol{S}^{1 \top}
$$

Note that Willis law is self-adjoint in the sense

$$
\boldsymbol{C}_{\boldsymbol{k}, \omega}^{e}=\boldsymbol{C}_{-\boldsymbol{k}, \omega}^{e \top}, \quad \boldsymbol{\rho}_{\boldsymbol{k}, \omega}^{e}=\boldsymbol{\rho}_{-\boldsymbol{k}, \omega}^{e \top} \quad \text { and } \quad \boldsymbol{S}_{\boldsymbol{k}, \omega}^{1}=\boldsymbol{S}_{-\boldsymbol{k}, \omega}^{2 \top} .
$$




\section{AppendixB. Expression of Q}

The denominator $Q$ of $Z$ from equation (6.1) is given by the expression

$$
\begin{aligned}
& 2 a \sqrt{c_{1} \rho_{1} c_{2} \rho_{2}}\left(c_{2} k^{2}-\rho_{2} \omega^{2}\right)\left(c_{1} k^{2}-\rho_{1} \omega^{2}\right)\left[\left(c_{1}+c_{2}\right) k^{2}-\left(\rho_{1}+\rho_{2}\right) \omega^{2}\right] \cos (2 k a) \\
& -4 \sqrt{c_{1} \rho_{1} c_{2} \rho_{2}}\left(c_{1} \rho_{2}-c_{2} \rho_{1}\right)\left[\left(c_{1}-c_{2}\right) k^{2}-\left(\rho_{1}-\rho_{2}\right) \omega^{2}\right] k \omega^{2} \sin (2 k a) \\
& +\sqrt{\rho_{2} c_{2}}\left[\sqrt{\rho_{1}}\left(c_{1}-c_{2}\right) k-\sqrt{c_{1}}\left(\rho_{1}-\rho_{2}\right) \omega\right]^{2} \omega \\
& \left\{\left(\sqrt{c_{1}} k+\sqrt{\rho_{1}} \omega\right)^{2} \sin \left[a\left(k+\sqrt{\rho_{1} / c_{1}} \omega\right)\right]\right. \\
& \left.-\left(\sqrt{c_{1}} k-\sqrt{\rho_{1}} \omega\right)^{2} \sin \left[a\left(k-\sqrt{\rho_{1} / c_{1}} \omega\right)\right]\right\} \\
& +\sqrt{c_{1} \rho_{1}}\left[\sqrt{\rho_{2}}\left(c_{1}-c_{2}\right) k-\sqrt{c_{2}}\left(\rho_{1}-\rho_{2}\right) \omega\right]^{2} \omega \\
& \left\{\left(\sqrt{c_{2}} k+\sqrt{\rho_{2}} \omega\right)^{2} \sin \left[a\left(k+\sqrt{\rho_{2} / c_{2}} \omega\right)\right]\right. \\
& \left.-\left(\sqrt{c_{2}} k-\sqrt{\rho_{2}} \omega\right)^{2} \sin \left[a\left(k-\sqrt{\rho_{2} / c_{2}} \omega\right)\right]\right\} \\
& +\frac{a}{2}\left(c_{1} k^{2}-\rho_{1} \omega^{2}\right)\left(c_{2} k^{2}-\rho_{2} \omega^{2}\right)\left[\left(c_{1}+c_{2}\right) k^{2}-\left(\rho_{1}+\rho_{2}\right) \omega^{2}\right] \\
& \left\{\left(\sqrt{c_{1} \rho_{1}}-\sqrt{c_{2} \rho_{2}}\right)^{2} \cos \left[\omega a\left(\sqrt{\rho_{2} / c_{2}}-\sqrt{\rho_{1} / c_{1}}\right)\right]\right. \\
& \left.-\left(\sqrt{c_{1} \rho_{1}}+\sqrt{c_{2} \rho_{2}}\right)^{2} \cos \left[\omega a\left(\sqrt{\rho_{2} / c_{2}}+\sqrt{\rho_{1} / c_{1}}\right)\right]\right\} \\
& -\sqrt{c_{1} \rho_{1} c_{2} \rho_{2}}\left[\left(c_{1}-c_{2}\right) k^{2}-\left(\rho_{1}-\rho_{2}\right) \omega^{2}\right]^{2} \omega \\
& \left\{\left(\sqrt{c_{1} \rho_{1}}+\sqrt{c_{2} \rho_{2}}\right) \sin \left[\omega a\left(\sqrt{\rho_{2} / c_{2}}+\sqrt{\rho_{1} / c_{1}}\right)\right]\right. \\
& \left.-\left(\sqrt{c_{1} \rho_{1}}-\sqrt{c_{2} \rho_{2}}\right) \sin \left[\omega a\left(\sqrt{\rho_{2} / c_{2}}-\sqrt{\rho_{1} / c_{1}}\right)\right]\right\} \\
& -\left(c_{1} \rho_{2}-c_{2} \rho_{1}\right)^{2} \omega^{3} k^{2} \\
& \left\{\left(\sqrt{c_{1} \rho_{1}}+\sqrt{c_{2} \rho_{2}}\right) \sin \left[\omega a\left(\sqrt{\rho_{2} / c_{2}}+\sqrt{\rho_{1} / c_{1}}\right)\right]\right. \\
& \left.+\left(\sqrt{c_{1} \rho_{1}}-\sqrt{c_{2} \rho_{2}}\right) \sin \left[\omega a\left(\sqrt{\rho_{2} / c_{2}}-\sqrt{\rho_{1} / c_{1}}\right)\right]\right\} .
\end{aligned}
$$

